\title{
1 Maturation signatures of conventional dendritic cell subtypes in COVID-19 reflect direct viral sensing

\footnotetext{
${ }^{1}$ Department of Biotechnology and Biosciences, University of Milano-Bicocca, Milan, Italy ${ }^{2}$ National Institute of Molecular Genetics "Romeo ed Enrica Invernizzi”, Milan, Italy

${ }^{3}$ Pediatric Department and Centro Tettamanti-European Reference Network PaedCan, EuroBloodNet, MetabERN-University of Milano-Bicocca-Fondazione MBBM-Ospedale, San Gerardo, Monza, Italy.

${ }^{4}$ ASST san Gerardo Hospital, Monza, Italy, School of Medicine and Surgery, University of Milano-Bicocca

${ }^{5}$ Laboratory of Medical Microbiology and Virology, Vita-Salute San Raffaele University, Milan, Italy.

${ }^{6}$ IRCCS San Raffaele Hospital, Milan, Italy.

${ }^{7}$ Department of Clinical Sciences and Community Health, University of Milano, Milan, Italy ${ }^{8}$ Institute for Quantitative and Computational Biosciences, University of California, Los Angeles
}

*Equal contribution

${ }^{\&}$ Corresponding author. Email: francesca.granucci@ unimib.it; granucci@ingm.org 
Abstract: Growing evidence suggests that conventional dendritic cells (cDCs) undergo aberrant maturation in COVID-19 and this negatively affects $\mathrm{T}$ cell activation. The presence of functional effector $\mathrm{T}$ cells in mild patients and dysfunctional $\mathrm{T}$ cells in severely ill patients suggests that adequate $\mathrm{T}$ cell responses are needed to limit disease severity. Therefore, understanding how cDCs cope with SARS-CoV-2 infections can help elucidate the mechanism of generation of protective immune responses. Here, we report that $\mathrm{cDC} 2$ subtypes exhibit similar infection-induced gene signatures with the up-regulation of interferon-stimulated genes and IL-6 signaling pathways. The main difference observed between DC2s and DC3s is the up-regulation of anti-apoptotic genes in DC3s, which explains their accumulation during infection. Furthermore, comparing cDCs between severe and mild patients, we find in the former a profound down-regulation of genes encoding molecules involved in antigen presentation, such as major histocompatibility complex class II (MHCII) molecules, $\beta_{2}$ microglobulin, TAP and costimulatory proteins, while an opposite trend is observed for proinflammatory molecules, such as complement and coagulation factors. Therefore, as the severity of the disease increases, $\mathrm{cDC} 2 \mathrm{~s}$ enhance their inflammatory properties and lose their main function, which is the antigen presentation capacity. In vitro, direct exposure of cDC2s to the virus recapitulates the type of activation observed in vivo. Our findings provide evidence that SARS-CoV-2 can interact directly with $\mathrm{cDC} 2 \mathrm{~s}$ and, by inducing the down-regulation of crucial molecules required for $\mathrm{T}$ cell activation, implements an efficient immune escape mechanism that correlates with disease severity.

65 


\section{Results and Discussion}

Clinical outcomes of COVID-19 are highly variable. Patients may show either no/mild symptoms (such as mild fever and cough) or severe respiratory involvement requiring hospitalization. In the most severe cases, Acute Respiratory Distress Syndrome (ARDS) can develop, with high levels of inflammatory hallmarks in the blood ${ }^{1,2}$ and diffuse intravascular coagulation (DIC) ${ }^{3,4}$. In a non-negligible number of cases, COVID-19 is lethal ${ }^{5}$. Patients presenting severe symptoms show immune dysregulation characterized by excessive release of type 1 and type 2 cytokines ${ }^{2}$, and alterations of lymphoid and myeloid populations in the peripheral blood ${ }^{6}$. Severe patients, diversely from mild patients, also show alterations in both Th17 and Th1 cell activation, with defects in the acquisition of effector functions ${ }^{7}$.

Cells of myeloid origin play a pivotal role during infections by sensing pathogens, producing inflammatory mediators and by contributing to the activation of adaptive immunity. In this context, dendritic cells (DCs) are particularly relevant since they are specialized in antigen presentation and $\mathrm{T}$ cell priming ${ }^{8}$. Given the functional specialization of DCs, the differences observed in the activated $\mathrm{T}$ cell compartments in severe versus mild patients suggest that alterations in activation may also be present in the conventional DC (cDC) compartment in patients presenting with different levels of disease severity. cDCs have been divided in two subtypes, $\mathrm{cDC} 1$ and $\mathrm{cDC} 2$, originating from a common precursor (pre-DCs) ${ }^{9,10,11,12}$. cDC1s have a high intrinsic capacity to cross-present antigens, due to the expression of the CLEC9A c-type lectin ${ }^{13}$, and activate CD8 ${ }^{+}$, Th1 and NK cells ${ }^{14}$. Myeloid cDC2s express different Pattern Recognition Receptors (PRRs) and can promote a wide range of immune responses and especially $\mathrm{CD} 4^{+} \mathrm{T}$ cell responses ${ }^{15}$. Recently, cDC2s have been divided in two subsets, DC2 and DC $3^{16,17,18}$. DC3s are a heterogeneous population and comprehend non inflammatory cells showing a CD163-CD14-CD5- phenotype, inflammatory $\mathrm{CD}_{163}{ }^{+} \mathrm{CD} 14^{+} \mathrm{CD}^{-}$cells and a $\mathrm{CD} 163^{+} \mathrm{CD} 14^{-} \mathrm{CD}^{-}$intermediate subpopulation 16.

112 Functional impairments of cDCs have been described in COVID-19 patients, with decreased

113 numbers in the blood ${ }^{19,20}$, but not in bronchoalveolar lavage (BAL) samples ${ }^{7}$, and reduced

114 functionality, in terms of cytokines production and T cell priming capacity when restimulated

115 in vitro ${ }^{21,22}$. Nevertheless, a defect of maturation following in vitro restimulation does not 116 necessarily indicate functional impairment, as activated DCs may not further respond to PRR 117 agonists. Therefore, no specific information is available concerning the impact of SARS-CoV1182 infection on the maturation of DC subtypes and a better understanding is mandatory given 
119 the specific role of cDC subtypes in the activation and skewing of adaptive immune responses 120 that ultimately contribute to COVID-19 pathogenesis ${ }^{23}$.

121 In seeking for the impact of SARS-CoV-2 infection on blood DC subtypes, we analyzed peripheral blood DCs from severe and mild COVID-19 patients, according to World Health

123 Organization (WHO) classification. Patients were enrolled from the STORM cohort (see

124 Supplementary Table 1 for the clinical data of the patients) of San Gerardo Hospital in 125 Monza, Italy.

126 cDC1s were identified as CLEC9A ${ }^{+}$and cDC2s as CD1c ${ }^{+} \mathrm{Fc} \varepsilon \mathrm{RI} \alpha^{+}$over the CD11 $\mathrm{c}^{+} \mathrm{MHCII}^{+}$ 127 PBMCs excluding cells expressing markers for $\mathrm{T}$ and B lymphocytes (CD3 and CD19, 128 respectively) and monocytes (CD88 and CD89) ${ }^{16}$. CD14 was included in the analysis to 129 identify DC3s ${ }^{17}$ (Supplementary Fig. S1 for gating strategies). Consistent with some studies

$130{ }^{20}$ but not others ${ }^{6}$, we found a decreasing trend in the frequency of cDC1s and DC2s and an 131 increasing trend in DC3s in COVID-19 patients compared with healthy donors (HDs) (Fig. 132 1A).

133 To perform a systematic characterization of the transcriptional response of DCs to SARS-CoV1342 infection, we analyzed three different single-cell transcriptomic datasets, two publicly 135 available and a newly generated one. The analysis of three independent datasets allowed us to 136 identify consistently altered signaling pathways, minimizing the effects of possible biases in 137 the single datasets.

138 The new dataset (dataset 1) was generated using a droplet-based single cell platform (10X

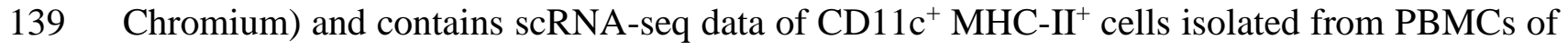
140 three COVID-19 patients (two mild and one severe) and two HDs (Supplementary Table 1). 141 The second dataset ${ }^{22}$ (dataset 2) contains cellular indexing of transcriptomes and epitopes by 142 sequencing (CITE-seq) data of PBMCs and enriched DCs obtained from 7 COVID-19 patients 143 (three mild and four severe) and 5 HDs, while the third dataset ${ }^{24}$ (dataset 3 ) contains scRNA144 seq data of PBMCs obtained from 18 COVID-19 patients (8 mild and 10 severe) and 21 HDs. 145 Single-cell data from datasets 1 and 2 were first visualized using non-linear dimensionality 146 reduction through uniform manifold approximation and projection (UMAP) and graph-based 147 clustering algorithms (Supplementary Fig. 2A, 3A). Clusters containing myeloid DCs were 148 identified based on the expression of markers that discriminate cDC2s and cDC1s from all 149 other cell populations. Specifically, CDIC, FCER1A and CLEC1OA were used to identify 150 cDC2s, while CLEC9A was used to identify cDC1s (Supplementary Fig. 2B, 3B). For dataset 1513 , myeloid DCs already annotated by the authors were considered ${ }^{24}$. Clusters corresponding 152 to myeloid DCs in the three datasets were re-clustered in further iterations to separate cDC1s 
153 from $\mathrm{cDC} 2 \mathrm{~s}$, to discriminate $\mathrm{cDC} 2$ subpopulations and to exclude possible contaminants. Specifically, DC3s were distinguished from DC2s based on the expression of CD14, CD163 and S100A8 markers. This approach allowed us to clearly identify cDC subsets (Fig. 1B and Supplementary Fig. 3C,D).

157 Next, in order to unravel the transcriptional response of each DC subset during SARS-CoV-2 infection, we aggregated cell-level counts into sample-level pseudobulk counts, mitigating single-cell mRNA measurement noise, and identified differentially expressed genes (DEGs)

160 between COVID-19 patients and HDs (Supplementary Table 2). The low numbers of cDC1s allowed their analysis only in dataset 2 .

In all DC subsets from the three datasets, when comparing expression profiles of COVID-19 patients with those of HDs, most of the genes up-regulated in COVID-19 were interferon (IFN) stimulated genes (ISGs) (Fig. 1C and Supplementary Fig. 4A,B). On the other hand, among the most significantly down-regulated genes in COVID-19, there were those encoding MHC class II molecules (Fig. 1C), indicating an impaired antigen presentation capacity of these cells. To understand more deeply which biological signaling pathways were differentially regulated in COVID-19 patients compared with HDs, we performed Gene Set Enrichment Analysis (GSEA) using two different gene sets: the Hallmark collection from the Molecular Signatures Database (MSigDB) and the literature-derived Blood Transcription Modules (BTMs) ${ }^{25}$ (Supplementary Table 3). As it could be predicted by the identified DEGs, in all DC subtypes from the three datasets, maturation was dominated by ISGs while we could not detect the upregulation of signatures containing classical activation markers and cytokines for $\mathrm{T}$ cell priming (Fig. 1D and Supplementary Fig. 5A). Together with the IFN-induced pathways, IL6 pathways (IL-6-JAK-STAT3 and PI3K-AKT-mTOR ${ }^{26}$ ) were recurrently up-regulated in cDC2s in all datasets (Fig. 1D). This is consistent with the relevance of IL-6 in COVID-19 pathogenesis and the expansion of activated Th17 cells in COVID-19 patients ${ }^{27}$.

The lack of a conventional maturation signature (lack of up-regulation of genes encoding MHCII and costimulatory molecules and cytokines) in circulating DCs prompted us to ask whether it was, in fact, possible to identify activated DCs in the blood. It could not be excluded that mature DCs reach circulation too late after activation when they are exhausted and, thus, only very late transcriptional events are visible.

We, therefore, investigated the transcriptional responses of circulating DC2 and DC3 subsets at single-cell resolution in different clinical conditions. Two distinct publicly available datasets were analyzed: the dataset from Reyes et al. ${ }^{28}$ containing scRNA-seq data of PBMCs and 
severity (localized infection [Leuk-UTI], systemic infection with transient [Int-URO] or persistent organ dysfunctions [URO]), and the dataset from Hao et al. ${ }^{29}$ containing CITE-seq data of PBMCs obtained from healthy volunteers that received an adenovirus-based vaccine. As previously described, we performed dimensionality reduction and unsupervised clustering to identify DC subpopulations. Our approach clearly identified DC subsets in both datasets (Fig. 2A and Supplementary Fig. 6). We then determined DEGs in infected or vaccinated

193 donors with respect to the corresponding HDs (Supplementary Table 4) and performed 194 GSEA.

195 The results were in stark contrast to those obtained from COVID-19 patients. Indeed, in both datasets, circulating DC2s and DC3s showed an up-regulation not only of IFN pathways (as in COVID-19) but also of inflammatory signatures and genes relevant for immune responses (differently from COVID-19) (Fig. 2B,C and Supplementary Fig. 7,8). Among the most highly up-regulated genes there were several encoding activation molecules such as CCRl, CCR5, CXCL10, TNFSF10 (CD253/TRAIL) as well as Toll-like receptor (TLR) genes, (Fig.

\section{B and Supplementary Fig. 7A,B).}

202

These findings were confirmed by pathway analysis, which showed a clear up-regulation of activation pathways in DC2 and DC3 subsets in response to bacterial infections or vaccine, such as the inflammatory response pathway and the TNF- $\alpha$ signaling pathway (Fig. $2 \mathrm{C}$ and Supplementary Fig. 8A,B). Among the leading edge genes driving the enrichment of the inflammatory response pathway in response to bacterial infections there were several ones relevant for $\mathrm{T}$ cell activation (IL1B, CCL5, TNFSF10, GPR183, CD69, SELL) (Supplementary Fig. 8C).

Interestingly, we observed a stronger activation response of circulating $\mathrm{cDC} 2 \mathrm{~s}$ in patients with localized bacterial infections (Leuk-UTI group) and transient organ dysfunction (Int-URO group) than in patients with bacterial sepsis and persistent organ dysfunction (URO group) (Fig. 2C). This was expected since sepsis induces functional impairment of myeloid cells.

213 These results indicate that conventionally activated DCs can be detectable in the blood when 214 infections are both localized and systemic. Therefore, the lack of a classic activation signature, 215 observed in cDC subtypes from blood of COVID-19 patients, is not a generalized phenomenon.

216 Recent studies have indicated potential functional differences between DC2 and DC3

217 subpopulations ${ }^{30}$, in particular in inflammatory diseases, like Systemic Lupus Erythematosus 218 (SLE), in which type I IFNs play a major role ${ }^{16}$. In order to investigate a potential specific role 219 for DC3s with respect to DC2s, we determined the genes differentially 
induced/downmodulated by these two subpopulations in response to SARS-CoV-2 stimulation and compared them to bacterial sepsis.

To increase our resolution, we pooled cDCs from the three COVID-19 datasets and performed Harmony integration ${ }^{31}$, followed by graph-based clustering. After integration, we obtained 2,415 cDCs (Fig. 3A) and we clearly identified clusters of cDC1s, DC2s and DC3s (Fig. 3B). Only 24 genes ( $\mathrm{p}$-value $<0.05$ and absolute $\log 2 \mathrm{FC}>1$ ) were differentially expressed in DC3s compared with DC2s in response to COVID-19 infection, of which 17 were up-regulated and 7 were down-regulated (Fig. 3C, left panel and Supplementary Table 5). On the other hand, 152 genes ( $\mathrm{p}$-value $<0.05$ and absolute $\log 2 \mathrm{FC}>1$ ) were identified as differentially regulated in DC3s compared with DC2s in response to intermediate urosepsis (Int-URO condition), of which 59 were up-regulated and 93 were down-regulated (Fig. 3C, right panel and Supplementary Table 5).

232 The diversity in the responses of DC3s and DC2s during bacterial infections could be, at least partly, explained by the differential expression of some receptors, such as CD14 exclusively expressed by DC3s. CD14 is a component of the receptor complex of lipopolysaccharide (LPS), a major factor of the outer membrane of Gram-negative bacteria, and contributes to LPS recognition and internalization of the receptor complex ${ }^{32}$. Therefore, thanks to the expression of CD14, DC3s can respond more efficiently to Gram-negative bacteria than DC2s. CD14 has also important roles as chaperon for ligands of endosomal and cytosolic PRRs ${ }^{32}$. Therefore, the differences between DC2 and DC3 responses observed in Gram-negative bacterial infections may also occur after Gram-positive bacterial recognition.

241 In conclusion, these findings suggest that DC2s and DC3s respond in a very similar way to SARS-CoV-2 infections, while they show more diversified responses to bacterial infections. Interestingly, among the few genes differentially expressed between DC3s and DC2s in response to COVID-19, there were genes encoding complement factors and receptors $(C 1 Q C$, $C 1 Q A$ and $C 5 A R 1)$ and, most importantly, anti-apoptotic genes such as $A X L$ and $C L U$ that resulted the most significantly up-regulated (Fig. 3C, left panel). This suggests that DC3s are less susceptible to apoptosis than DC2s and may explain why they tend to increase while all other cDC populations decrease during SARS-CoV-2 infection (Fig. 1A). When comparing these results with those obtained from bacterial infections, we found that genes associated with cell cycle progression and cell proliferation (RGCC, SENP5, SMC6, SERTAD3, MAD2L1BP) were specifically up-regulated in DC3s (Fig. 3C, right panel). Therefore, DC3s may proliferate during inflammatory responses or circulating DC3s may contain some proliferating progenitors that expand the DC3 population during bacterial infections. 
254 Altogether, these observations could explain why DC3s increase in number in acute and chronic inflammatory conditions ${ }^{16,33}$. Moreover, the higher persistence potential of DC3s induced by inflammation could explain why their frequency is highly variable in HDs.

257 Coherently with these observations, we found an alteration of cDCs relative abundance in COVID-19 patients compared with HDs also at single-cell resolution. Specifically, DC3s showed increased frequencies in patients, which positively correlated with disease severity (Fig. 3D). Since DC3s are highly heterogeneous, we analyzed DC3 subpopulations at higher resolution. Hence, we retained clusters corresponding to DC3s and performed a re-clustering procedure to discriminate between DC3s and inflammatory DC3s (Fig. 3E). Interestingly, we identified three main phenotypes, characterized by low expression of both CD14 and CD163 (clusters 2 and 6), intermediate expression (clusters 0, 1, 7 and 8) and high expression (clusters $3,4,5)$, reflecting the heterogeneity of DC3 population (Fig. 3F,G,H). Clusters with the highest expression of both CD14 and CD163 (clusters 3, 4, 5) were annotated as inflammatory DC3s. We found a progressive increase in the relative abundance of inflammatory DC $3 \mathrm{~s}$ from HDs, to mild and finally to severe patients (Fig. 3I). This result was associated with the higher expression of the anti-apoptotic gene $C L U$ in the inflammatory DC3s population, specifically in critically ill patients (Fig. 3J,K). Accordingly, an increasing trend in the frequency of inflammatory DC3s $\left(\mathrm{CD} 14^{+} \mathrm{CD} 163^{+}\right)$, and not of non-inflammatory DC3s (CD14-CD163-), was observed in the blood of severe patients (Fig. 3L).

In order to seek for specific alterations in the innate immune signature of mild and severe COVID-19 patients and to link immune response variation to disease severity, we investigated cDC2 gene expression profiles in severe versus mild COVID-19 patients. As previously described, we aggregated cell-level counts into sample-level pseudo-bulk counts and identified DEGs between severe and mild COVID-19 patients (Supplementary Table 6). In both DC2s and DC3s, we identified an important number of DEGs (200 for DC2s and 169 for DC3s, p-value < 0.05 and absolute $\log 2 \mathrm{FC}>1$ ) between severe and mild patients, indicating relevant differences in the transcriptional response of these two groups (Fig. 4A). Interestingly, inflammatory genes not directly related to the activation of adaptive immunity, like complement factors $(C 1 Q C, C 1 Q B)$ and complement receptors $(C 5 A R I)$, genes involved in the production of leukotrienes known to exacerbate respiratory syndromes (ALOX5AP), genes of the coagulation cascade (THBS1,THBD), factors involved in vasodilation $(A D M)$ and other inflammatory genes like $C D 14, S 100 A 8 / A 9, A D A M 9$ and $C D 163$ were significantly upregulated in severe versus mild patients in DC2s and/or DC3s (Fig. 4A). In addition, genes that 
negatively interfere with the maturation of DCs finalized to T cell activation, like TMEM176A, CD109, MT1E were up-regulated in severe compared to mild patients (Fig. 4A). Moreover, further supporting what discussed above, the anti-apoptotic gene $C L U$ was found to be among the most significantly up-regulated genes in DC3s of severe patients (Fig.4A, right panel).

291 Strikingly, genes encoding MHCII molecules, the costimulatory molecule CD86 and 292 cytokines, such as $I L 1 B, C C L 3$ and $C C L 4$, showed a progressive down-regulation from HDs 293 to mild and finally severe patients (Fig. 4B).

294 These observations were consolidated by pathway analysis, which showed a clear up295 regulation of pathways involved in metabolism, coagulation, angiogenesis and reactive oxygen species in severe compared with mild patients, and a down-regulation of IFN pathways (Fig. 4C). Interestingly, among the leading edge genes of the allograft rejection pathway, that was found to be down-regulated in severe compared with mild patients, there were many genes critical for DC-mediated T cell activation, such as those coding for proteins involved in antigen presentation on both MHCI and MHCII pathways (B2M, TAP1, TAP2, HLA-DMB, HLA-DRA) and genes encoding molecules relevant for $\mathrm{T}$ cell recruitment and activation $(I L 16, I L 1 B$, CCL4) (Fig. 4D). Specific down-regulation of these genes in severely ill patients emphasizes the alteration of $\mathrm{cDC}$ functions in these individuals, which may be associated with a worse disease progression.

Altogether, these observations indicate that, as disease severity increases, cDC2s progressively skew toward inflammatory activities and lose the antigen presenting function. This could explain the alteration of the activated T cell compartment observed in severely ill patients.

Results shown till now indicate that, during COVID-19 infections, DC3s and DC2s respond similarly to the virus with three main features: i) the up-regulation of ISGs and IL-6 pathways; ii) a progressive down-regulation from mild to severe patients of genes encoding signal 1 and signal 2 molecules associated with antigen presentation; iii) the up-regulation of an inflammatory signature, mainly represented by complement and coagulation factors, in severe 313 patients.

314 We wondered whether these features could be due to the exposure to mediators released during 315 SARS-CoV-2 infection or to the direct interaction of cDCs with the virus. The first cDC 316 characteristic observed in our study is compatible with both the direct interaction with the virus 317 and the exposure to paracrine cytokines, such as IFNs and IL-6 produced by bystander cells.

318 The lack of expression of IFN and IL-6 genes in circulating DCs does not necessarily mean 319 that cDCs cannot be a source of these cytokines, since the expression of genes encoding these molecules is acutely regulated and may be shut down when DCs reach the circulation. By 
contrast, the systematic down-regulation of genes encoding MHCII molecules is more likely explained by a direct interaction of $\mathrm{cDCs}$ with the virus. This prediction was also supported by evidence that the virus can directly activate monocyte derived DCs following abortive infection 34.

325 Therefore, we investigated whether the direct interaction of $\mathrm{cDC} 2 \mathrm{~s}$ with the virus could induce a similar response to that observed at single-cell resolution. By using IL-6 and MHCII as readouts, we measured the response to the virus of $\mathrm{cDC} 2 \mathrm{~s}\left(\mathrm{CD} 1 \mathrm{c}^{+} \mathrm{CD} 19^{-}\right.$cells $)$freshly isolated from HDs. As predicted, we found that SARS-CoV-2 directly induced a significant downregulation of MHCII surface expression and the up-regulation of IL-6 in both DC2s and DC3s (Fig. 5A,B). Diversely, the exposure of $\mathrm{cDC} 2 \mathrm{~s}$ to sera from mild and severe patients, that contain inflammatory cytokines and other mediators, could not induce any modification in MHCII and IL-6 expression (Fig. 5C). This suggests that at least part of the peculiar response of cDCs induced in vivo by SARS-CoV-2 infection can be directly imposed by the virus.

334 In conclusion, SARS-CoV-2 can be detected directly by DCs and induces down-regulation of signals necessary for activation of $\mathrm{T}$ lymphocytes, a phenomenon that is accentuated with disease severity. This allows the virus to evade control of the adaptive immune system, while the host attempts to counteract viral infection with innate immunity. Understanding how DCs manage SARS-CoV-2 infection will help identify ad hoc interventions to achieve optimal adaptive responses, a prerequisite for a good prognosis ${ }^{35,23}$. 


\section{Materials and Methods}

350

351

352

353

354

355

356

357

358

359

360

361

362

363

364

365

366

367

368

369

370

371

372

373

374

375

376

377

378

379

\section{Flow cytometric analysis}

PBMCs from COVID-19 patients enrolled from the STORM cohort were extracted from peripheral blood by density gradient centrifugation using Ficoll (GE Healthcare). Cells were washed twice and stained for 30 minutes on ice using the following anti-human antibodies (1:200, Becton Dickinson): anti-FceRIa PE-Cy7, anti-CD14 PE, anti-CD1c APC-Cy7, antiClec9 (CD370) Alexa 647, anti-CD5 BV786, anti-CD3 BV605, anti-CD19 BV605, anti-CD88 BV605, anti-CD89 BV605, anti-CD11c BV480, anti-CD163 BV421, anti-HLA-DR BUV805. Cells were then washed and fixed using fixation buffer (Becton Dickinson) and acquired using BD FACSsymphony instrument (Becton Dickinson). Analyses were performed with Flow jo X software.

\section{cDC2s purification and activation}

Human cDC2 cells were purified from peripheral blood mononuclear cells (PBMCs) extracts from buffy coat of healthy donors (provided by Niguarda hospital blood bank) by Ficoll-Paque density gradient centrifugation. Briefly, blood was stratified on Ficoll-Paque PLUS (GE Healthcare) in 3:4 ratio and centrifuged at 1500 r.p.m. for 30 min without brake. PBMCs were washed twice, collected and CD1 $\mathrm{c}^{+}$cells were purified using MACS beads according to the manufacturer's instructions (Miltenyi Biotec). Cells were cultured in Roswell Park Memorial Institute (RPMI) 1640 medium (Euroclone) containing 10\% heat-inactivated fetal bovine serum (Euroclone), $100 \mathrm{IU}$ of penicillin, streptomycin $(100 \mu \mathrm{g} / \mathrm{ml}), 2 \mathrm{mM}$ l-glutamine (Euroclone). cDC2s were infected with $0.4 \mathrm{MOI}$ of SARS-CoV-2 for $18 \mathrm{~h}$ or treated with serum of COVID-19 patients (ratio serum/medium 1:1), then collected and stained with anti-FceRIa PE-Cy7, anti-CD14 PE, anti-CD1c APC-Cy7, anti-CD5 BV786, anti-CD3 BV605, anti-CD19 BV605, anti-CD88 BV605, anti-CD89 BV605, anti-CD11c BV480, anti-CD163 BV421, antiHLA-DR BUV805 (1:200, all from Becton Dickinson). Cells were then fixed and permeabilized with cytofix/cytoperm reagent kit (Becton Dickinson) and stained with anti-IL6 FITC antibody, according to the manufacturer's instructions. Samples were acquired with the BD FACSsymphony instrument (Becton Dickinson) and analyzed with Kaluza software.

\section{Single-cell RNA sequencing datasets analyzed in the study}

In this study, three different single-cell datasets from COVID-19 patients and healthy controls were analyzed. 
380 Dataset 1 was newly generated. Myeloid cells were sorted (CD11 $\left.\mathrm{c}^{+} \mathrm{MHCII}^{+}\right)$from 3 COVID38119 patients ( 2 mild and 1 severe, enrolled from the STORM cohort) and 2 healthy donors using 382 a MACSQuant Tyto (Miltenyi) (Supplementary Fig. 1B). After sorting, cell number and 383 viability were evaluated using an automated cell counter. Viability for each sample was $\geq 75 \%$. 384 10,000 cells per sample were loaded on a Chromium Next GEM Chip G (10x Genomics). A 385 Chromium controller (10x Genomics, Pleasanton, CA, USA) was used to generate single-cell 386 GEMs, according to Chromium Next GEM Single Cell 5' Library \& Gel Bead Kit v1.1 387 protocol (PN-1000165; 10x Genomics). Full-length cDNA amplification and 5' gene expression library construction were performed according to manufacturers' instructions in a Veriti 96-well Thermal Cycler (Thermo Fisher Scientific). Indexed libraries were sequenced on an Illumina Novaseq 6000 platform, on a S2 flowcell, 150bp PE (20,000 read pairs per cell). Reads from FASTQ files were aligned against the GRCh38 human reference genome and quantified using the Cell Ranger pipeline (10x Genomics) version 3.0 with default parameters.

393 Single cell data have been deposited in GEO (GSE168388) and will be accessible upon 394 publication.

395 Dataset $2^{22}$ is a CITE-seq experiment with PBMCs and enriched DCs from 7 COVID-19 396 patients (three mild and four severe) and 5 HDs. Count matrices were downloaded from the 397 Gene Expression Omnibus (GEO) (GSE155673).

398 Dataset $3^{24}$ is a scRNA-seq experiment with PBMCs from 18 COVID-19 patients (8 mild and 39910 severe) and 21 HDs. Seurat objects were downloaded from FASTGenomics 400 (https://www.fastgenomics.org/). Only cells annotated as myeloid DCs by the authors were 401 used in downstream analyses.

402 To compare transcriptional responses of cDC subsets between SARS-CoV-2 infection and 403 other inflammatory conditions, we analyzed two additional publicly available datasets.

404 The Reyes et al. dataset ${ }^{28}$ is a scRNA-seq experiment with PBMCs and enriched DCs obtained 405 from patients with bacterial infections and healthy controls. Briefly, subjects were enrolled in 406 two different cohorts. A primary cohort contains subjects that were classified into three clinical 407 categories: Leuk-UTI, Int-URO and URO. The Leuk-UTI group refers to subjects with urinary408 tract infection (UTI) with leukocytosis (blood $\mathrm{WBC} \geq 12,000$ per $\mathrm{mm}^{3}$ ) but no organ 409 dysfunction. The Int-URO (intermediate urosepsis) group contains subjects with UTI with mild 410 or transient organ dysfunction, and the URO (urosepsis) group refers to subjects with UTI with 411 clear or persistent organ dysfunction. Ten subjects were classified as Leuk-UTI, seven as Int412 URO and ten as URO. A second cohort comprises hospitalized subjects classified into three 413 conditions: subjects with bacteremia and sepsis not requiring intensive care unit (ICU) 
414 admission (Bac-SEP group, four subjects), subjects with sepsis requiring ICU care (ICU-SEP,

415 eight subjects) and subjects in the ICU for conditions other than sepsis (ICU-NoSEP, seven

416 subjects). Data were downloaded from the Broad Institute Single Cell Portal

417 (https://singlecell.broadinstitute.org/single_cell) (SCP548). For downstream analysis, we

418 retained monocytes and DCs as annotated by the authors.

419 The Hao et al. dataset ${ }^{29}$ is a CITE-seq experiment with PBMCs from 8 healthy volunteers 420 enrolled in an adenovirus-based HIV vaccine trial. For each subject, PBMCs were collected at 421 three time points: immediately before (day 0), three days, and seven days following vaccine administration. Data were downloaded from https://atlas.fredhutch.org/nygc/multimodalpbmcl. For downstream analysis, we retained only cDCs as annotated by the authors.

\section{Single-cell data processing and analysis}

425 Data processing and analysis for all single-cell datasets was performed using the Seurat package (version 4.0) ${ }^{29}$ in $\mathrm{R}$ (version 4.0.3).

427 First, filters were applied to remove low-quality cells. These were based on the number of genes and UMIs detected in each cell and on the percentage of reads mapping to mitochondrial genes (cells with $<500$ genes and $>10 \%$ of reads mapping to mitochondrial RNA were removed). Counts were then normalized and log-transformed using sctransform ${ }^{36}$, while regressing out UMI counts and percentage of mitochondrial counts.

432 For dimensionality reduction, PCA was performed. Principal components (PCs) were fed to 433 Harmony ${ }^{31}$ for batch correction and/or integration of datasets from both disease and healthy conditions. UMAP was used for 2D visualization. Clusters were identified with the shared nearest neighbour (SNN) modularity optimization-based clustering algorithm followed by Louvain community detection. Cell type assignment was manually performed using marker genes, as detailed in figures. cDCs were retained and re-clustered again to identify subsets.

\section{Pseudobulk differential gene expression analysis}

439 After the identification of cDC subsets, we aggregated cell-level counts into sample-level

440 pseudobulk counts. For each DC subset, only donors with at least 10 cells were retained.

441 For the dataset from Reyes et al., only samples from the primary cohort were considered for

442 differential analysis due to the low number of DCs obtained from subjects from the secondary 443 cohort.

444 Differential expression analysis was performed using the quasi-likelihood framework of the edgeR package ${ }^{37}$, using each donor as the unit of independent replication. 


\section{Gene Set Enrichment Analysis}

448 Pre-ranked GSEA ${ }^{38}$ was performed on the differentially expressed genes (DEGs) using the

449 fgsea package ${ }^{39}$. The Hallmark gene sets and the Blood Transcription Modules (BTM) ${ }^{25}$ were

450 used. BTM families analyzed in this study are reported in Supplementary Table 3.

\section{Integration between datasets}

452 cDCs identified in the three COVID-19 datasets were pooled, integrated using Harmony ${ }^{31}$,

453 and further subclustered using the shared nearest neighbour (SNN) modularity optimization-

454 based clustering algorithm followed by Louvain community detection with a resolution of 0.6

455 to identify cDC1, DC2 and DC3 clusters.

\section{Code availability}

457 Code used for data analysis will be made available upon publication.

458

459

460

461

462

463

464

465

466

467

468

469

470

471

472

473

474

475

476

477

478

479 


\section{References}

481 1. Silvin, A. et al. Elevated Calprotectin and Abnormal Myeloid Cell Subsets Discriminate Severe from Mild COVID-19. Cell (2020) doi:10.1016/j.cell.2020.08.002.

2. Lucas, C. et al. Longitudinal analyses reveal immunological misfiring in severe COVID-19. Nature (2020) doi:10.1038/s41586-020-2588-y.

3. Mangalmurti, N. \& Hunter, C. A. Cytokine Storms: Understanding COVID-19. Immunity (2020) doi:10.1016/j.immuni.2020.06.017.

4. Merrill, J. T., Erkan, D., Winakur, J. \& James, J. A. Emerging evidence of a COVID19 thrombotic syndrome has treatment implications. Nature Reviews Rheumatology (2020) doi:10.1038/s41584-020-0474-5.

Wu, Z. \& McGoogan, J. M. Characteristics of and Important Lessons from the Coronavirus Disease 2019 (COVID-19) Outbreak in China: Summary of a Report of 72314 Cases from the Chinese Center for Disease Control and Prevention. JAMA Journal of the American Medical Association (2020) doi:10.1001/jama.2020.2648.

6. Laing, A. G. et al. A dynamic COVID-19 immune signature includes associations with poor prognosis. Nat. Med. (2020) doi:10.1038/s41591-020-1038-6.

7. Wauters, E. et al. Discriminating mild from critical COVID-19 by innate and adaptive immune single-cell profiling of bronchoalveolar lavages. Cell Res. 31, 272-290 (2021).

8. Cabeza-Cabrerizo, M., Cardoso, A., Minutti, C. M., Pereira da Costa, M. \& Reis e Sousa, C. Dendritic Cells Revisited. Annu. Rev. Immunol. (2021) doi:10.1146/annurevimmunol-061020-053707.

9. Eisenbarth, S. C. Dendritic cell subsets in T cell programming: location dictates function. Nat. Rev. Immunol. 19, 89-103 (2019).

10. See, P. et al. Mapping the human DC lineage through the integration of highdimensional techniques. Science (80-. ). 356, (2017).

11. Anderson, D. A., Dutertre, C. A., Ginhoux, F. \& Murphy, K. M. Genetic models of human and mouse dendritic cell development and function. Nature Reviews Immunology (2020) doi:10.1038/s41577-020-00413-X.

12. Guilliams, M. et al. Unsupervised High-Dimensional Analysis Aligns Dendritic Cells across Tissues and Species. Immunity (2016) doi:10.1016/j.immuni.2016.08.015.

13. Canton, J. et al. The receptor DNGR-1 signals for phagosomal rupture to promote cross-presentation of dead-cell-associated antigens. Nat. Immunol. (2021) doi:10.1038/s41590-020-00824-x.

14. Jongbloed, S. L. et al. Human CD141+(BDCA-3)+ dendritic cells (DCs) represent a unique myeloid DC subset that cross-presents necrotic cell antigens. J. Exp. Med. (2010) doi:10.1084/jem.20092140.

15. Nizzoli, G. et al. Human CD1c+ dendritic cells secrete high levels of IL-12 and potently prime cytotoxic T-cell responses. Blood (2013) doi:10.1182/blood-2013-04495424.

16. Dutertre, C. A. et al. Single-Cell Analysis of Human Mononuclear Phagocytes Reveals Subset-Defining Markers and Identifies Circulating Inflammatory Dendritic Cells. Immunity 51, 573-589.e8 (2019). 
17. Villani, A. C. et al. Single-cell RNA-seq reveals new types of human blood dendritic cells, monocytes, and progenitors. Science (80-. ). 356, (2017).

18. Cytlak, U. et al. Differential IRF8 Transcription Factor Requirement Defines Two Pathways of Dendritic Cell Development in Humans. Immunity (2020) doi:10.1016/j.immuni.2020.07.003.

19. Sánchez-Cerrillo, I. et al. COVID-19 severity associates with pulmonary redistribution of CD1c+ DCs and inflammatory transitional and nonclassical monocytes. J. Clin. Invest. (2020) doi:10.1172/JCI140335.

20. Kvedaraite, E. et al. Major alterations in the mononuclear phagocyte landscape associated with COVID-19 severity. Proc. Natl. Acad. Sci. (2021) doi:10.1073/pnas.2018587118.

21. Zhou, R. et al. Acute SARS-CoV-2 Infection Impairs Dendritic Cell and T Cell Responses. Immunity (2020) doi:10.1016/j.immuni.2020.07.026.

22. Arunachalam, P. S. et al. Systems biological assessment of immunity to mild versus severe COVID-19 infection in humans. Science (80-. ). 369, 1210-1220 (2020).

23. Sette, A. \& Crotty, S. Adaptive immunity to SARS-CoV-2 and COVID-19. Cell (2021) doi:10.1016/j.cell.2021.01.007.

24. Schulte-Schrepping, J. et al. Severe COVID-19 Is Marked by a Dysregulated Myeloid Cell Compartment. Cell (2020) doi:10.1016/j.cell.2020.08.001.

25. Li, S. et al. Molecular signatures of antibody responses derived from a systems biology study of five human vaccines. Nat. Immunol. (2014) doi:10.1038/ni.2789.

26. Pullamsetti, S. S., Seeger, W. \& Savai, R. Classical IL-6 signaling: A promising therapeutic target for pulmonary arterial hypertension. Journal of Clinical Investigation (2018) doi:10.1172/JCI120415.

27. De Biasi, S. et al. Marked T cell activation, senescence, exhaustion and skewing towards TH17 in patients with COVID-19 pneumonia. Nat. Commun. 11, 3434 (2020).

28. Reyes, M. et al. An immune-cell signature of bacterial sepsis. Nature Medicine (2020) doi:10.1038/s41591-020-0752-4.

29. Hao, Y. et al. Integrated analysis of multimodal single-cell data. bioRxiv (2020) doi:10.1101/2020.10.12.335331.

30. Bourdely, P. et al. Transcriptional and Functional Analysis of CD1c+ Human Dendritic Cells Identifies a CD163+ Subset Priming CD8+CD103+ T Cells. Immunity 53, 335-352.e8 (2020).

31. Korsunsky, I. et al. Fast, sensitive and accurate integration of single-cell data with Harmony. Nat. Methods (2019) doi:10.1038/s41592-019-0619-0.

32. Zanoni, I. \& Granucci, F. Role of CD14 in host protection against infections and in metabolism regulation. Front. Cell. Infect. Microbiol. 4, (2013).

33. Bakdash, G. et al. Expansion of a BDCA1+ CD14+ myeloid cell population in melanoma patients may attenuate the efficacy of dendritic cell vaccines. Cancer Res. (2016) doi:10.1158/0008-5472.CAN-15-1695.

34. Zheng, J. et al. Severe Acute Respiratory Syndrome Coronavirus 2-Induced Immune Activation and Death of Monocyte-Derived Human Macrophages and Dendritic Cells. J. Infect. Dis. (2020) doi:10.1093/infdis/jiaa753. 
35. Tan, A. T. et al. Early induction of functional SARS-CoV-2-specific T cells associates with rapid viral clearance and mild disease in COVID-19 patients. Cell Rep. (2021) doi:10.1016/j.celrep.2021.108728.

36. Hafemeister, C. \& Satija, R. Normalization and variance stabilization of single-cell RNA-seq data using regularized negative binomial regression. Genome Biol. (2019) doi:10.1186/s13059-019-1874-1.

37. Robinson, M. D., McCarthy, D. J. \& Smyth, G. K. edgeR: A Bioconductor package for differential expression analysis of digital gene expression data. Bioinformatics (2009) doi:10.1093/bioinformatics/btp616.

38. Subramanian, A. et al. Gene set enrichment analysis: A knowledge-based approach for interpreting genome-wide expression profiles. Proc. Natl. Acad. Sci. U. S. A. (2005) doi:10.1073/pnas.0506580102.

39. Sergushichev, A. A. An algorithm for fast preranked gene set enrichment analysis using cumulative statistic calculation. bioRxiv (2016). 
605 Acknowledgments: RS thanks the QCB Collaboratory community directed by Matteo 606 Pellegrini.

607

608 Funding: F.G. is supported by Fondazione Cariplo (INNATE-CoV), Fondazione Veronesi 609 (FRACOVID), AIRC (IG 2019Id.23512), Fondazione Regionale per la Ricerca Biomedica, 610 FRRB (IANG-CRC - CP2_12/2018), and Ministero della Salute, Ricerca Finalizzata (RF611 2018-12367072).

612

613 Author contributions:

614 Conceptualization: FG, LM

615 Methodology: LM, RS, GP, VR, ARP, LS, VB, CS, MCC, SA

616 Investigation: LM, GP, RS, FAF, MV, FM, SA, SC, MDA, LB, AB, LN, NT

617 Visualization: FG, GP, LM

618 Funding acquisition: FG

619 Supervision: FG

620 Writing - original draft: FG, GP

621 Writing - review \& editing: FG, GP, LM

622 Competing interests: RS is currently an employee of GlaxoSmithKline. The other authors 623 declare that they have no competing interests.

624 Data and materials availability: All data are available in the main text or in the supplementary 625 materials.

\section{Supplementary Materials}

627 Figs. S1 to S8

628 Tables S1 to S6

629

630

631

632

633

634

635

636

637

638

639

640

641

642

643

644 
647 Fig. 1. The response of cDCs to SARS-CoV-2 infection is dominated by ISGs. (A) 648 Percentage of $\mathrm{cDC} 1 \mathrm{~s}$, DC2s and DC3s on CD45 ${ }^{+}$cells from whole blood of COVID-19 patients 649 ( $\mathrm{n}=22$ mild and $\mathrm{n}=10$ severe $)$ and HDs $(\mathrm{n}=21)$. Statistical significance was determined using 650 one-way analysis of variance, followed by Sidak's multiple comparison test. ${ }^{*} \mathrm{p}<0.05 ; *$; $<$ 651 0.01. (B, upper panels) UMAP representations of $\mathrm{cDC}$ subtypes identified from the three 652 scRNA-seq datasets analysed: dataset 1 (newly generated) and datasets 2 and 3 (publicly 653 available). Cells are colored according to cDC subtype and donor origin (pink, HDs; lightblue, 654 COVID-19). (B, lower panels) Violin plots illustrating expression levels of selected marker genes used for the manual annotation of cDC subtypes. (C) Heatmaps showing the top 100 DEGs for cDC1, DC2 and DC3 subsets comparing COVID-19 patients and HDs from dataset 2. Selected up-regulated genes (ISGs) are marked in red and down-regulated genes in blue. Ribosomal protein (RP) genes were removed from the top 100 DEGs. (D) GSEA of DEGs using the Hallmark collection: dataset 1 (left panel), dataset 2 (middle panel) and dataset 3 (right panel). For each DC subset, top 15 pathways based on significance are shown. NES, normalized enrichment score.

Fig. 2. Activation signature of cDCs during bacterial infections and adenovirus-based vaccine administration. (A) UMAP representations of $\mathrm{cDC}$ subtypes and corresponding violin plots illustrating expression levels of selected marker genes used for the manual annotation of cDC subtypes: Reyes et al. dataset (left panel) and Hao et al. dataset (right panel). (B) Heatmaps showing the top 100 DEGs for DC2 and DC3 subsets comparing: Leuk-UTI patients with HDs from Reyes et al. dataset (left panel) and vaccinated donors at day 3 with unvaccinated donors from Hao et al. dataset (right panel). Selected up-regulated genes are marked in red. Asterisk indicates genes associated with pro-inflammatory functions. Ribosomal protein (RP) genes were removed from the top 100 DEGs. (C) GSEA of DEGs using the Hallmark collection: Reyes et al. dataset (left panel) and Hao et al. dataset (right panel). For each DC subset, top 10 pathways based on significance are shown. NES, normalized enrichment score. Leuk-UTI, urinary tract infection with leukocytosis. Int-URO, intermediate urosepsis. URO, urosepsis. DC3s accumulate in severe patients. (A) UMAP representations of cDCs after datasets integration. Cells are colored according to dataset origin (left panel; pink, dataset 1; green, 
679 patients; lightblue, severe patients). (B) UMAP representation of cDC subtypes and 680 corresponding violin plots illustrating expression levels of selected marker genes used for the manual annotation of cDC subtypes. (C) Volcano plots showing genes differentially induced in DC3s compared with DC2s in response to COVID-19 (left panel) and intermediate urosepsis (Int-URO condition, Reyes et al. dataset, right panel). Genes with p-value $<0.05$ and absolute $\log _{2}$ fold change $>1$ were considered significant. Selected genes are highlighted (red: up in DC3s; blue: up in DC2s). (D) Barplots showing the relative abundance of cDC populations in HDs, mild and severe patients. (E) Subclustering of DC3 population to identify inflammatory DC3s (clusters 3, 4 and 5). (F-H) Expression levels of selected marker genes used for the identification of inflammatory DC3s within the DC3 population: $(\mathrm{F})$ violin plots showing expression levels of HLA-DRB1, CLEC10A, CLEC9A, CD14,CD163 and S100A8, (G) dotplot showing expression levels of CD14 and CD163 across DC3 clusters and (H) combined feature plot demonstrating co-expression of CD14 and CD163 in clusters 3, 4 and 5. (I) Barplots showing the relative abundance of DC3s and inflammatory DC3s in HDs, mild and severe patients. (J-K) Expression level of the anti-apoptotic gene CLU in DC3s and inflammatory DC3s is shown as $(\mathrm{J})$ dotplot and $(\mathrm{K})$ split violin plot by clinical condition (pink, HDs; blu, mild patients; lightblue, severe patients). (L) Percentage of inflammatory $\left(\mathrm{CD} 14^{+} \mathrm{CD} 163^{+}\right.$) and non-inflammatory (CD14-CD163) $\mathrm{DC} 3 \mathrm{~s}$ on $\mathrm{CD} 45^{+}$cells from whole blood of COVID-19 patients ( $\mathrm{n}=22$ mild and $\mathrm{n}=10$ severe) and HDs $(\mathrm{n}=21)$. capacity in severe COVID-19 patients. (A) Volcano plots showing genes differentially expressed in severe compared with mild patients in DC2s (left panel) and DC3s (right panel). Genes with p-value $<0.05$ and absolute $\log _{2}$ fold change $>1$ were considered significant. Boxplots showing expression levels of selected genes in DC2s and DC3s in HDs, mild and severe patients. Statistical analyses were performed using Wilcoxon rank sum test. $* \mathrm{p}<0.05$; $* * \mathrm{p}<0.01$. (C) GSEA of DEGs in severe compared with mild patients using the Hallmark collection. For each DC subset, top 10 pathways based on significance are shown. NES, normalized enrichment score. (D) Heatmaps showing leading edge genes of the allograft rejection pathway in mild and severe patients. Asterisk indicates genes associated with fundamental functions for DC-mediated T cell activation. 
712 expression in cDC2s from HDs infected or not (NT) with 0.4 MOI of SARS-CoV-2 for 18

713 hours. DC2s and DC3s were identified as $\mathrm{CD}^{+} \mathrm{CD}^{-c^{+}}$and $\mathrm{CD}^{-} \mathrm{CD} 1 \mathrm{c}^{+}$respectively over the

$714 \mathrm{CD}^{+}{ }^{+} \mathrm{LIN}^{-}\left(\mathrm{CD} 88, \mathrm{CD} 89, \mathrm{CD} 3\right.$ and CD19) and FceRIa ${ }^{+}$. (A, lower panel) Quantitative

715 analysis of mean fluorescence intensity (MFI) of HLA-DR in DC2s and DC3s. Statistical

716 significance was determined with unpaired student's t-test. $* \mathrm{p}<0.05, * * \mathrm{p}<0.01 ; \mathrm{n}=4 \mathrm{NT}$

717 donors and $\mathrm{n}=7$ donors for SARS-CoV-2 infection. (B, upper panel) Representative dot plots

718 showing the percentage of IL-6 producing DC2s and DC3s after viral infection as described in

719 B. (B, lower panel) Quantitative analysis of the percentage of IL-6 producing cells. Statistical

720 significance was determined with unpaired student's t-test. **p $<0.01 ; n=4$ NT donors and

$721 \mathrm{n}=7$ donors for SARS-CoV-2 infection. (C, left panel) Quantitative analysis of the percentage

722 of IL-6 producing DC2s and DC $3 \mathrm{~s}$ after $18 \mathrm{~h}$ incubation with sera from $\mathrm{n}=4$ mild and $\mathrm{n}=4$ severe

723 COVID-19 patients, NT (not treated). (C, right panel) Quantitative analysis of mean

724 fluorescence intensity (MFI) of HLA-DR in DC2s and DC3s treated or not (NT) for 18h with

725 sera from $n=4$ mild and $n=4$ severe COVID-19 patients. 
A
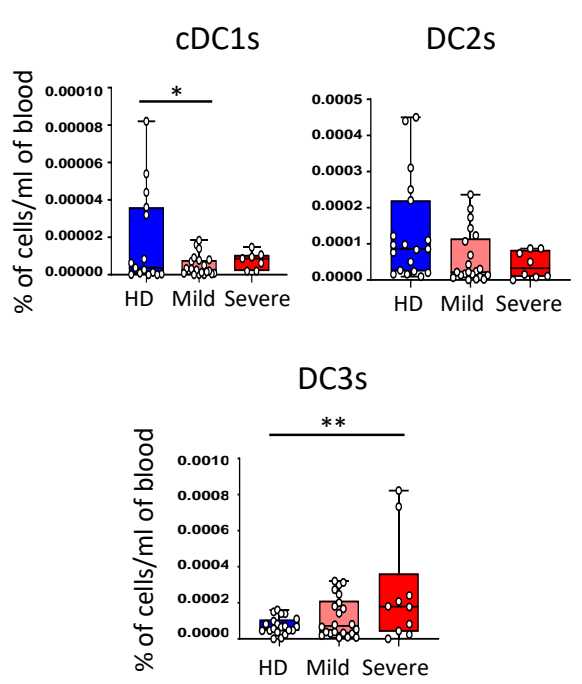

C

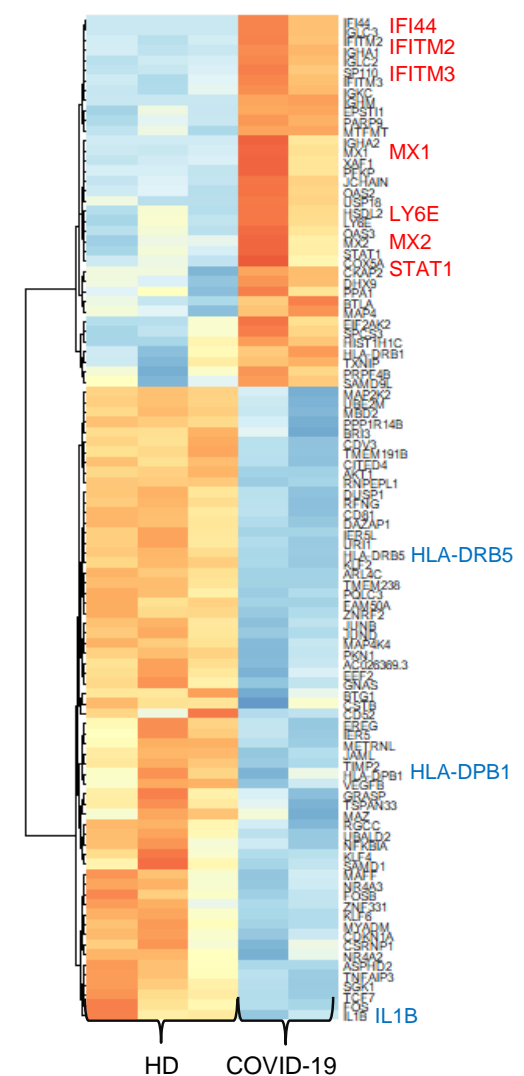

D

INTERFERON ALPHA RESPONSE - $\bullet$ INTERFERON GAMMA RESPONSE OXIDATIVE PHOSPHORYLATION PI3K AKT MTOR SIGNALING ALLOGRAFT REJECTION COMPLEMENT NOTCH SIGNALING HEME METABOLISM ANGIOGENESIS KRAS SIGNALING UP UV RESPONSE UP APOPTOSIS

INFLAMMATORY RESPONSE IL2 STAT5 SIGNALING P53 PATHWAY ESTROGEN RESPONSE LATE KRAS SIGNALING DN HYPOXIA

ESTROGEN RESPONSE EARLY TNFA SIGNALING VIA NFKB
B

Dataset 1

Dataset 2

- HD

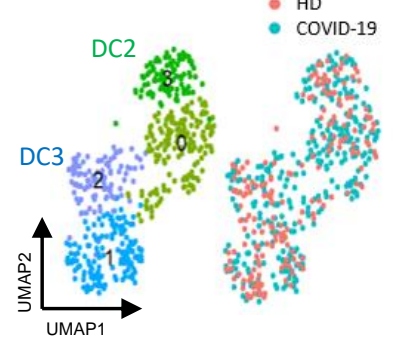

离 HLA-DRB1 ब CLEC10A $\bar{\Phi}$ CLEC9A
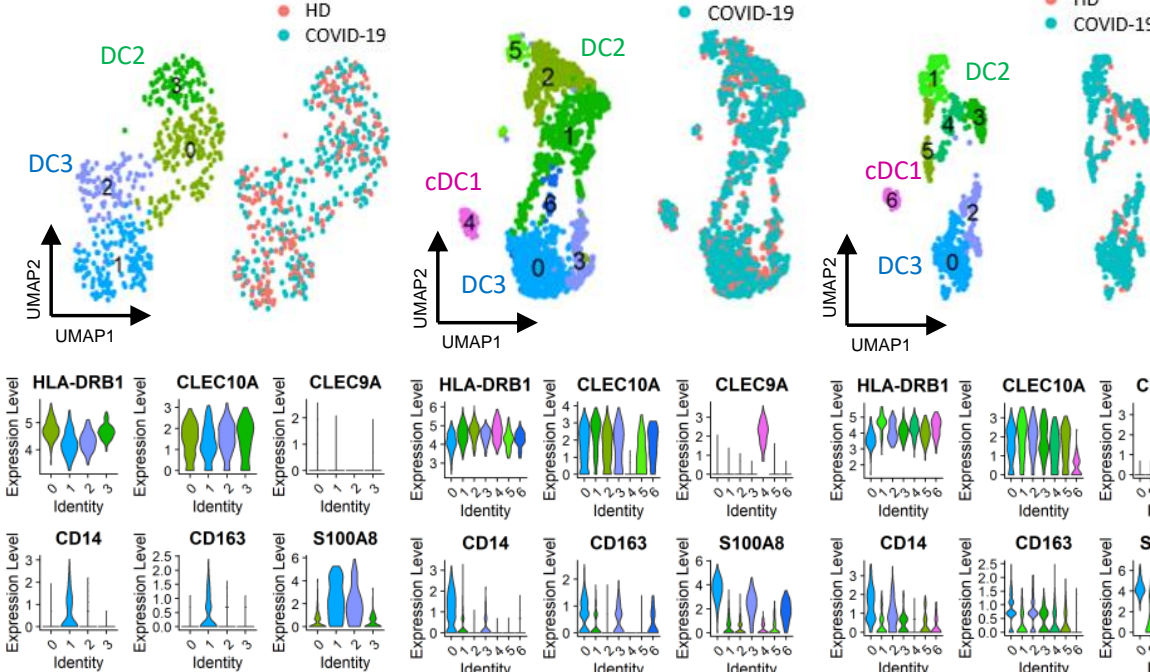

HD

COVID-19

Dataset 3

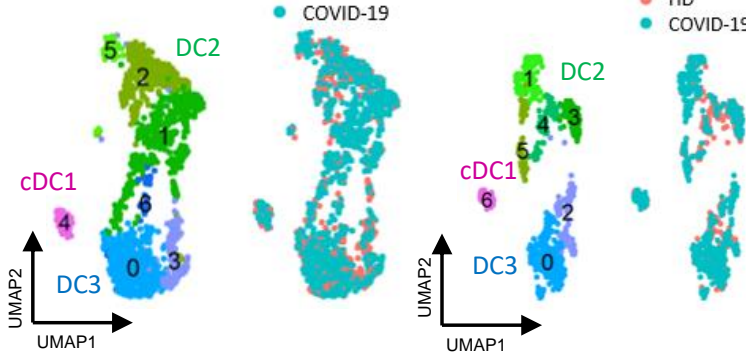

ब HLA-DRB1 ब CLEC10A ब CLEC9A
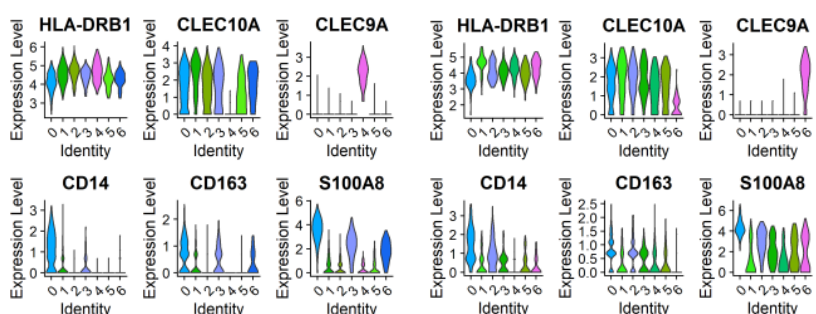

Dataset 2: DC2s

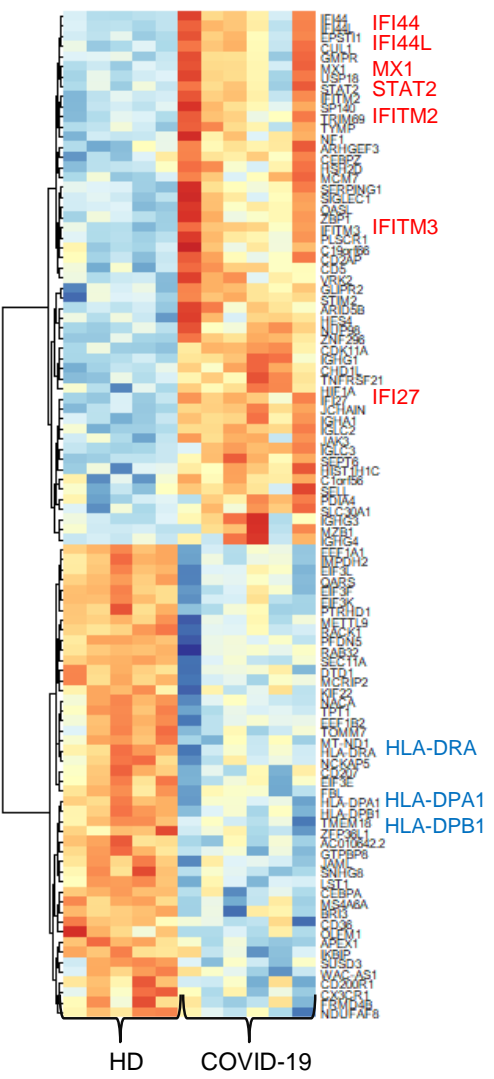

Dataset 2

INTERFERON ALPHA RESPONSE TERFERON GAMMA RESPONSE

888

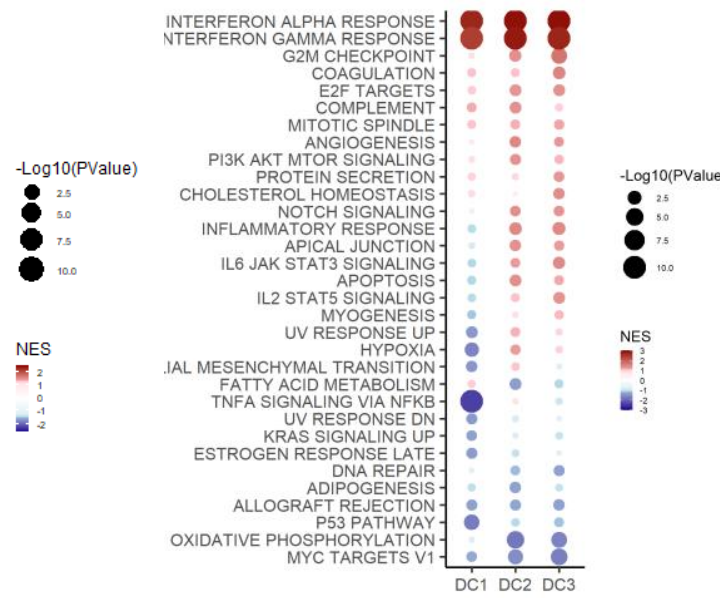

Dataset 2: DC3s

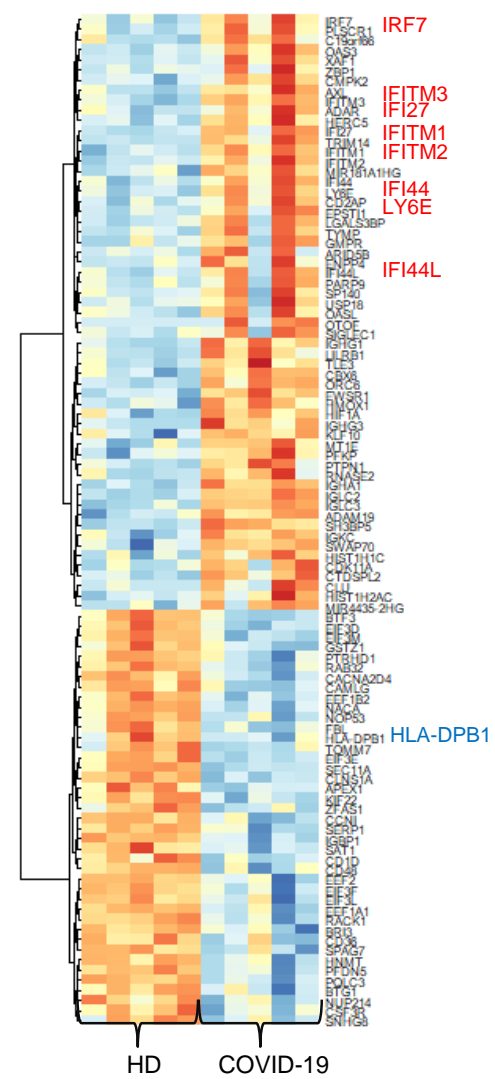

Figure 1

Dataset 3

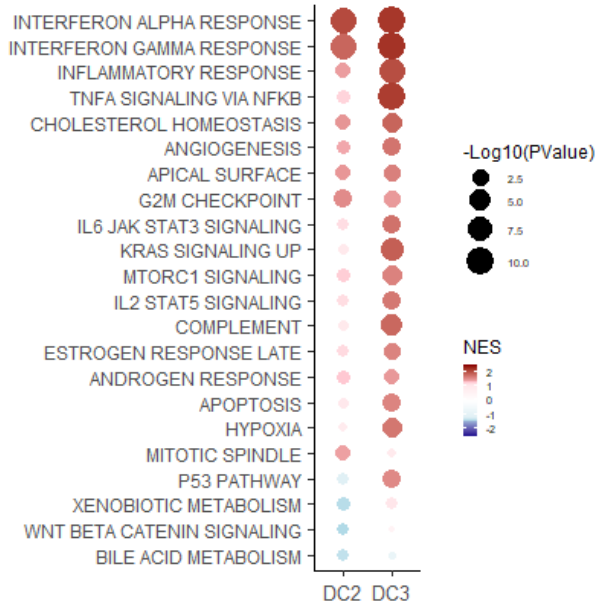




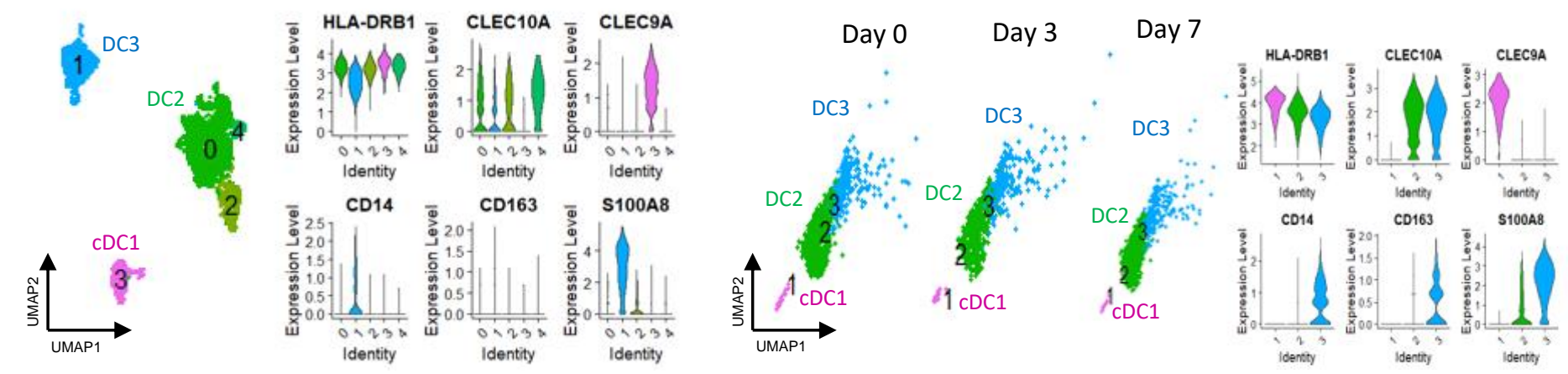

B

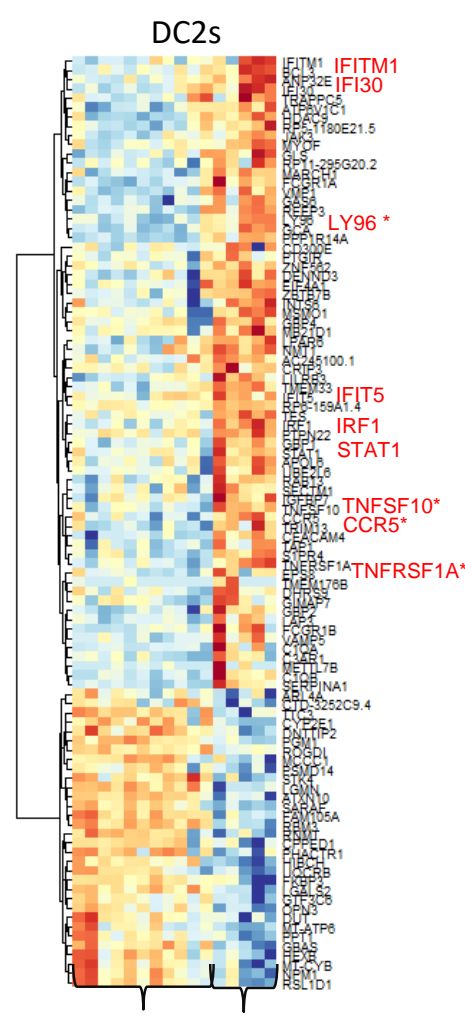

HD Leuk-UTI
Reyes et al. dataset

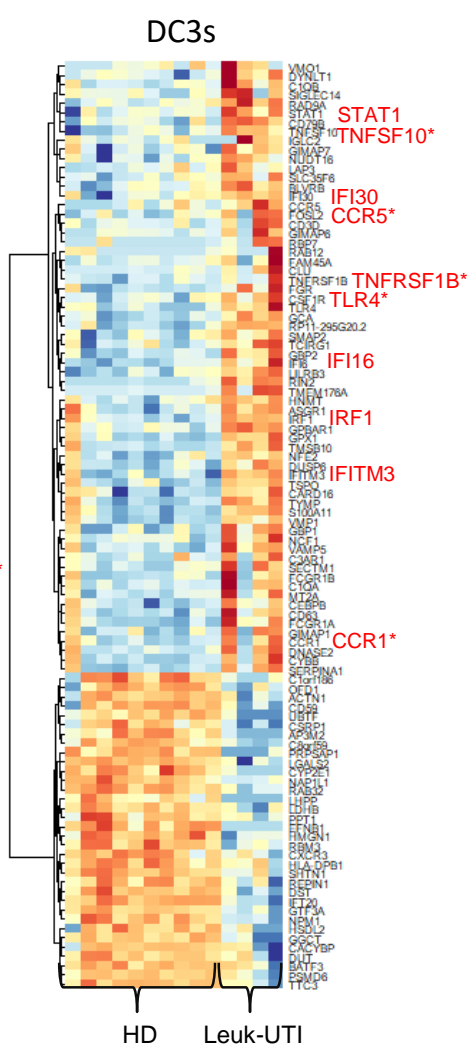

Reyes et al. dataset

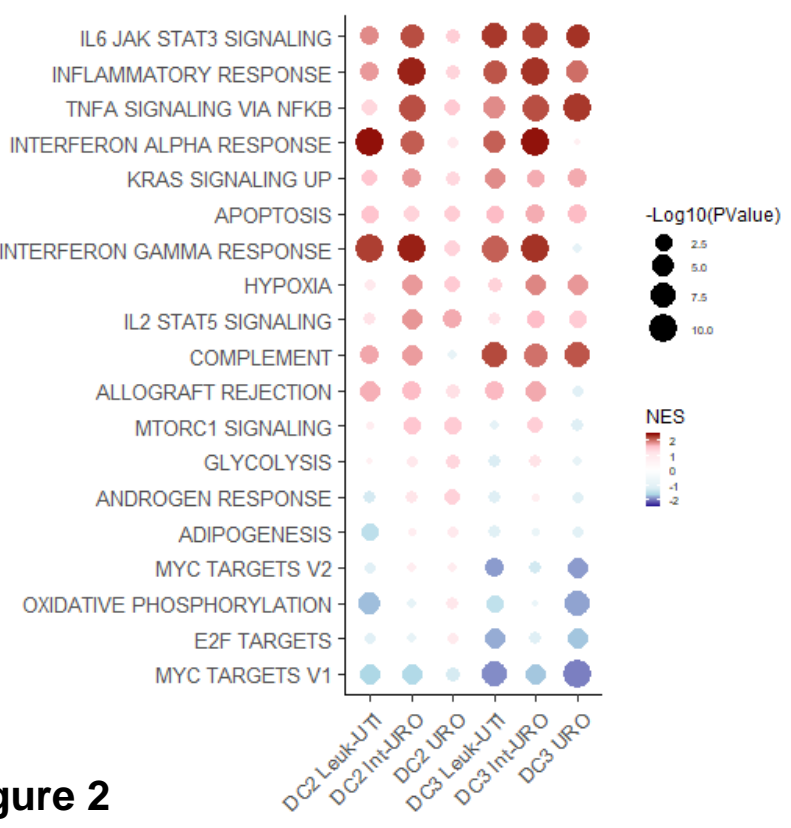

Hao et al. dataset

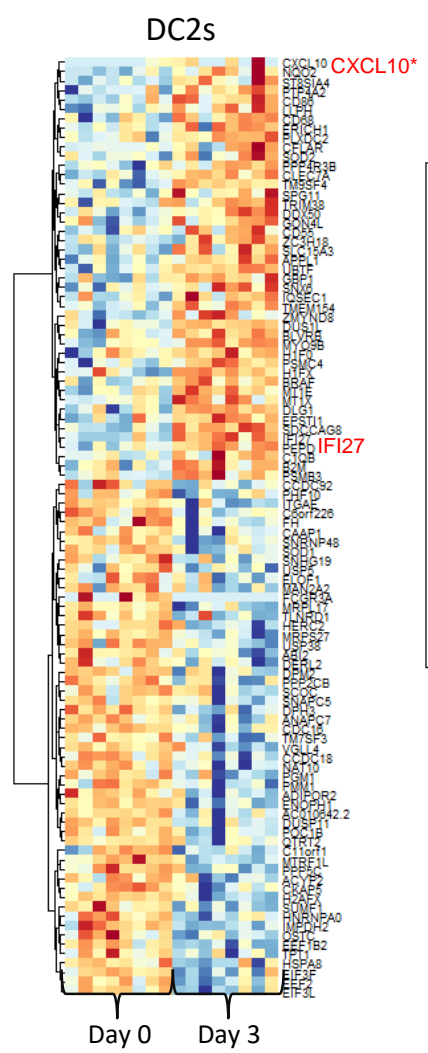

C

Figure 2
Hao et al. dataset

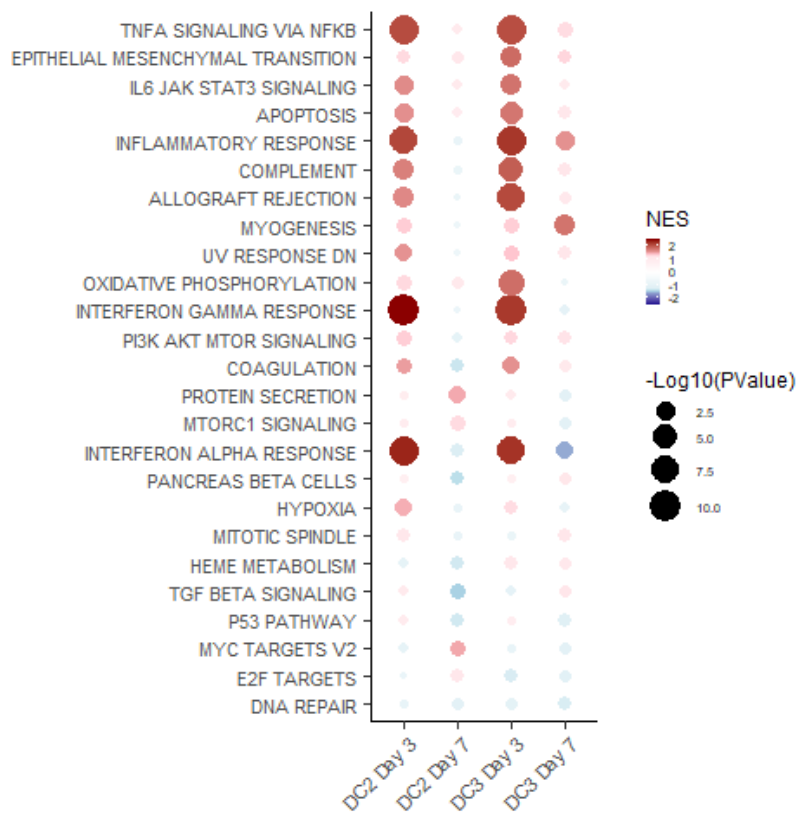


A $2,415 \mathrm{cDCs}$
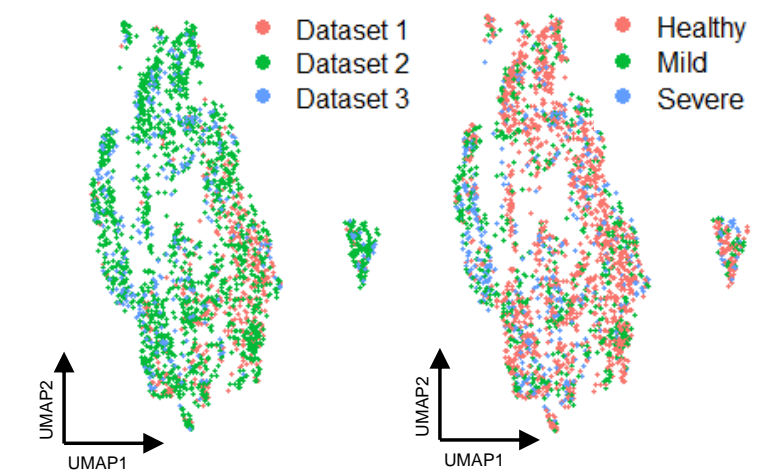

B

C
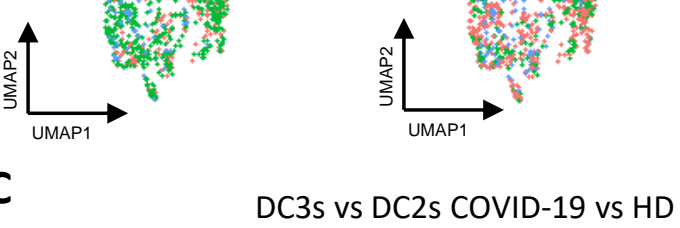
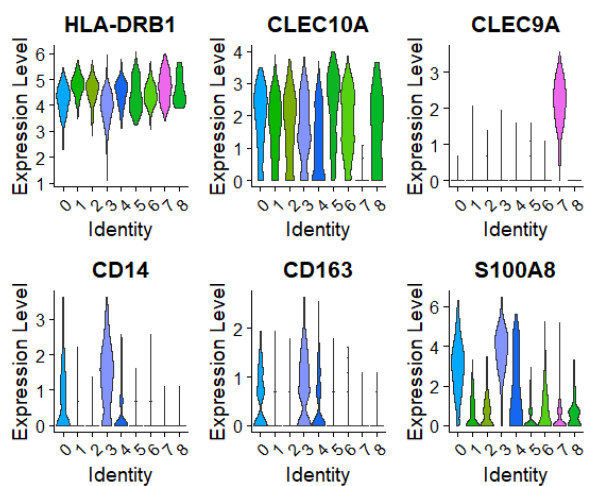

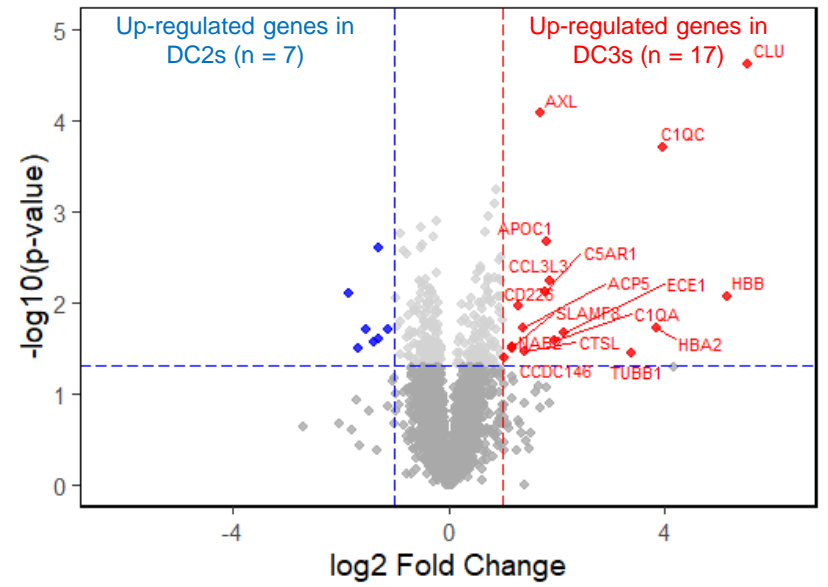

D

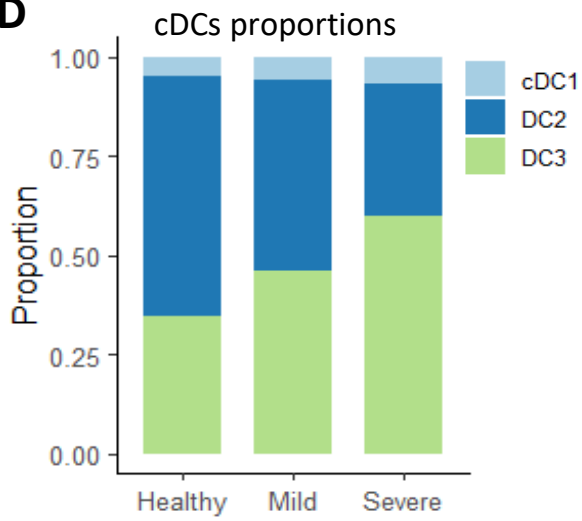

G

E

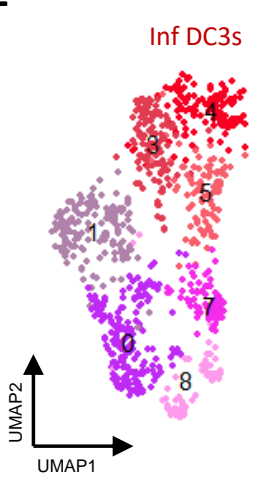

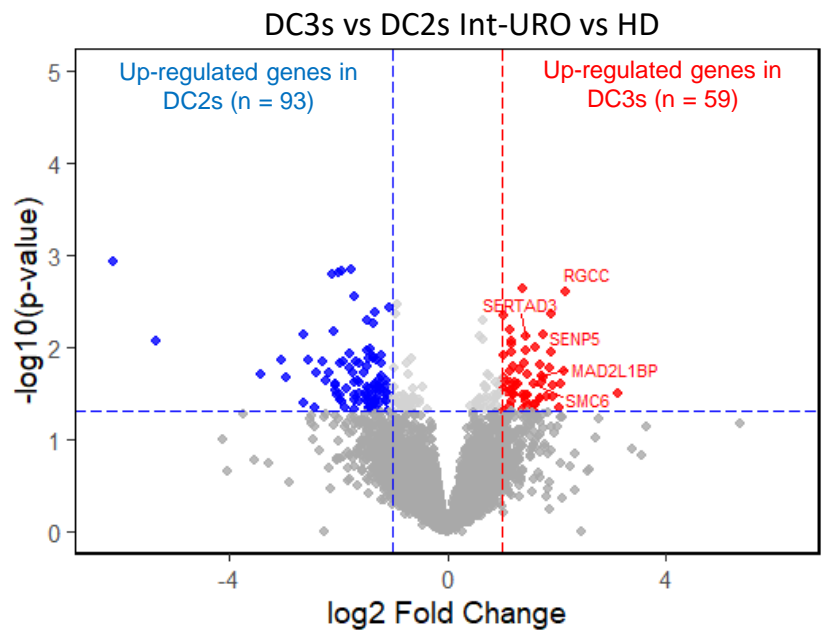

$\mathbf{F}$

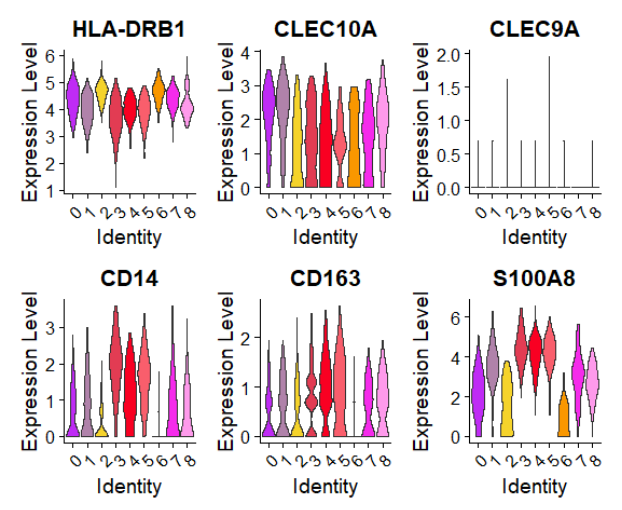

H

I DC3s and Inf DC3s proportions

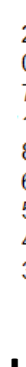

8

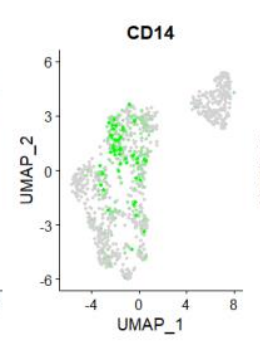

K

Figure 3

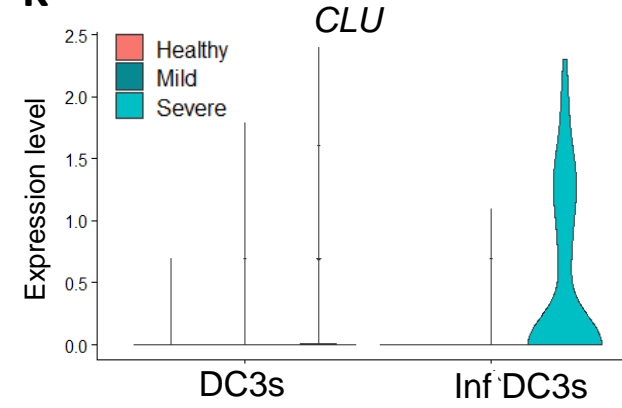

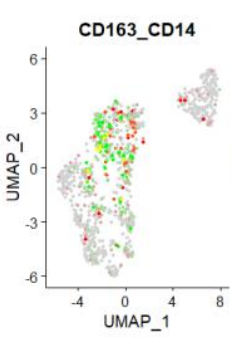

L
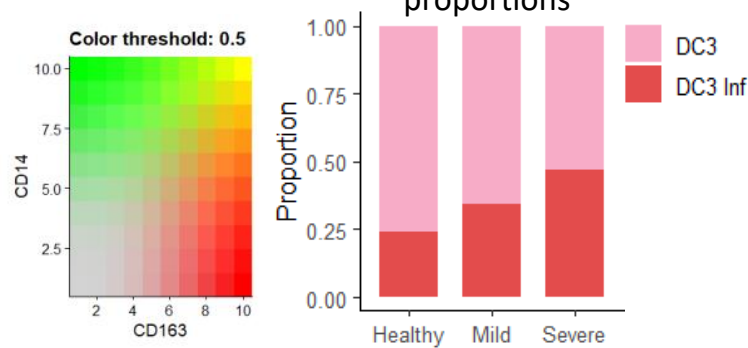

$\mathrm{CD}_{163}{ }^{+} \mathrm{CD} 14^{+}$

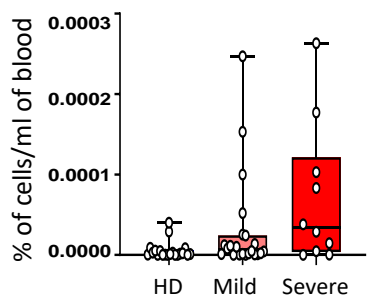

CD163-CD14-

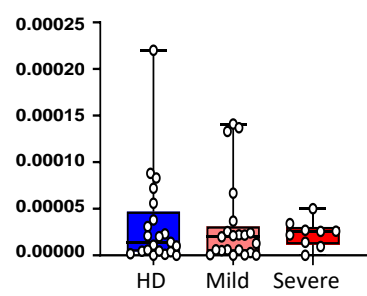


A

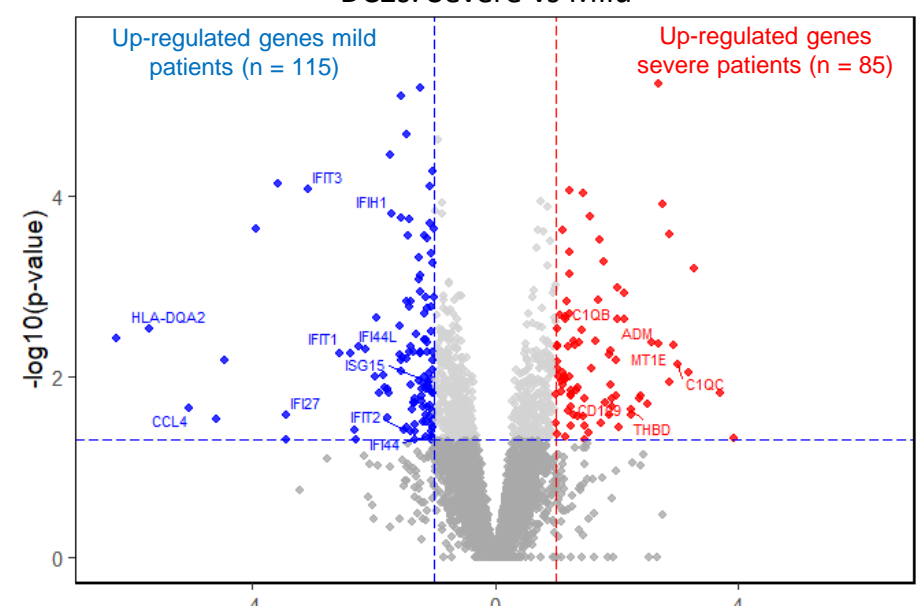

log2 Fold Change

\section{DC3s: Severe vs Mild}

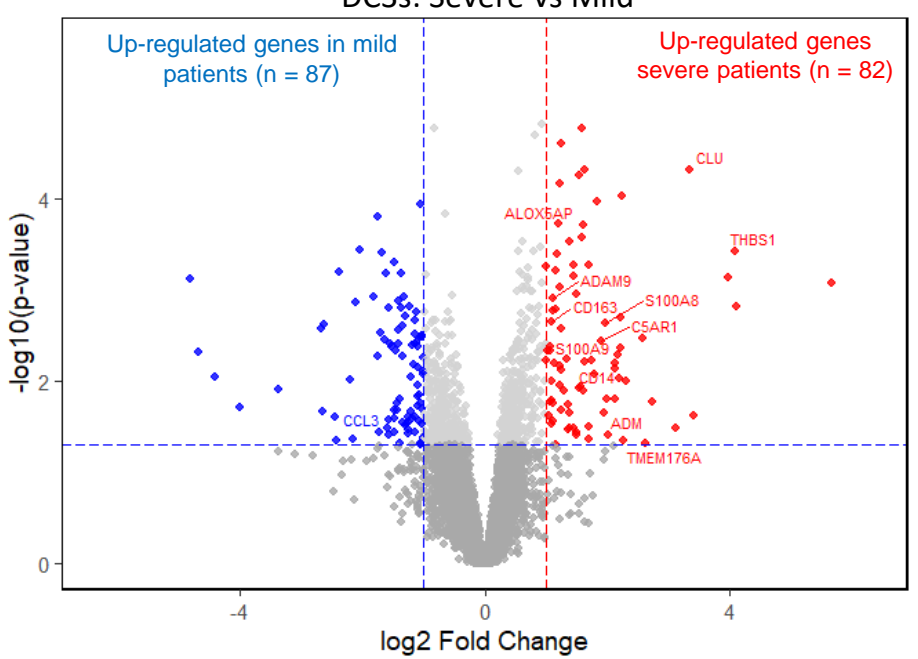

B DC2s
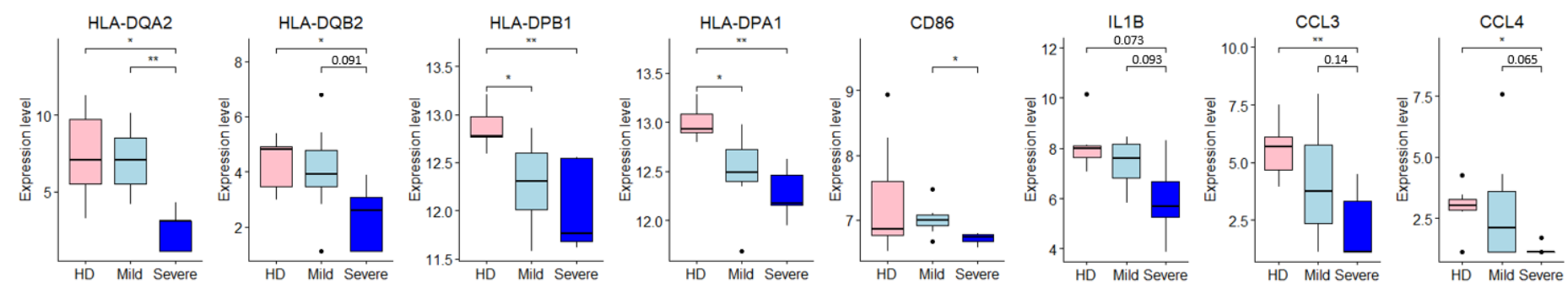

\section{DC3s}
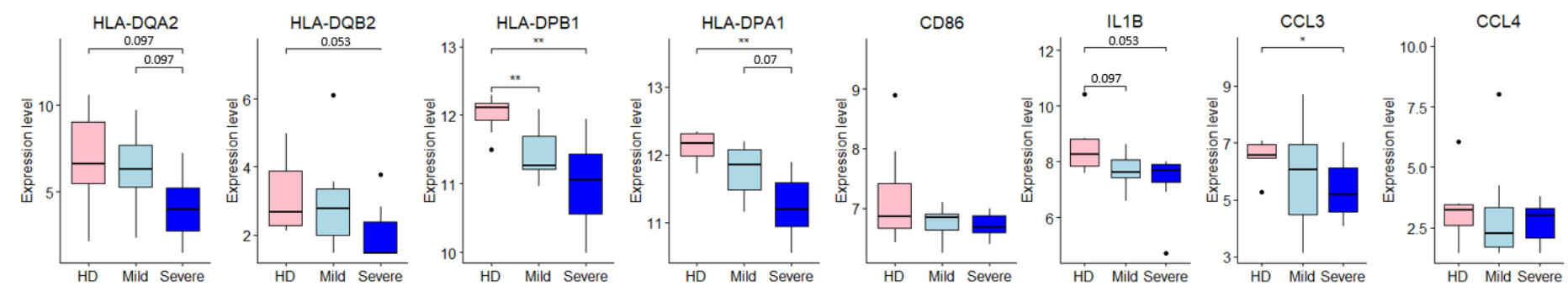

C

\section{GSEA: Severe vs Mild}

D
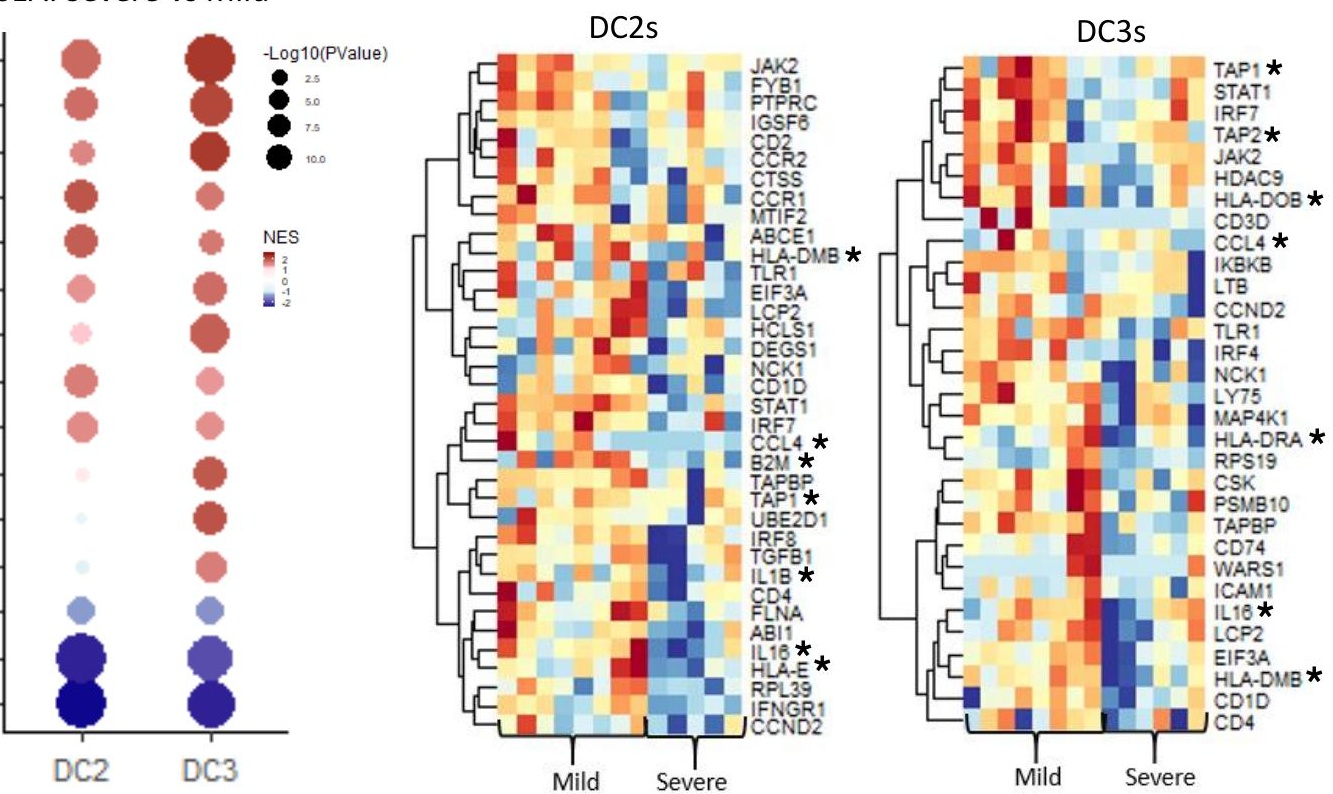

Figure 4 


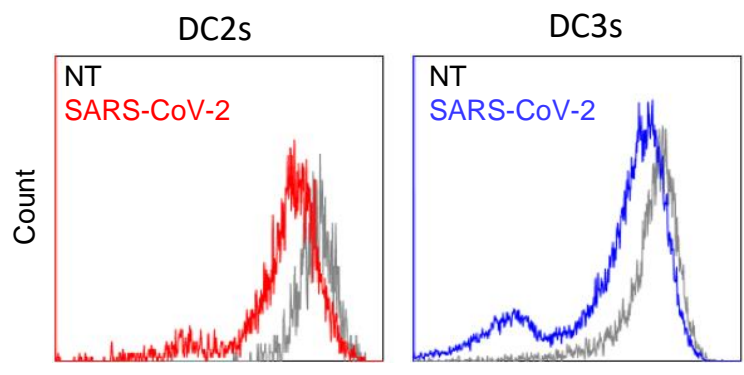

HLA-DR

HLA-DR
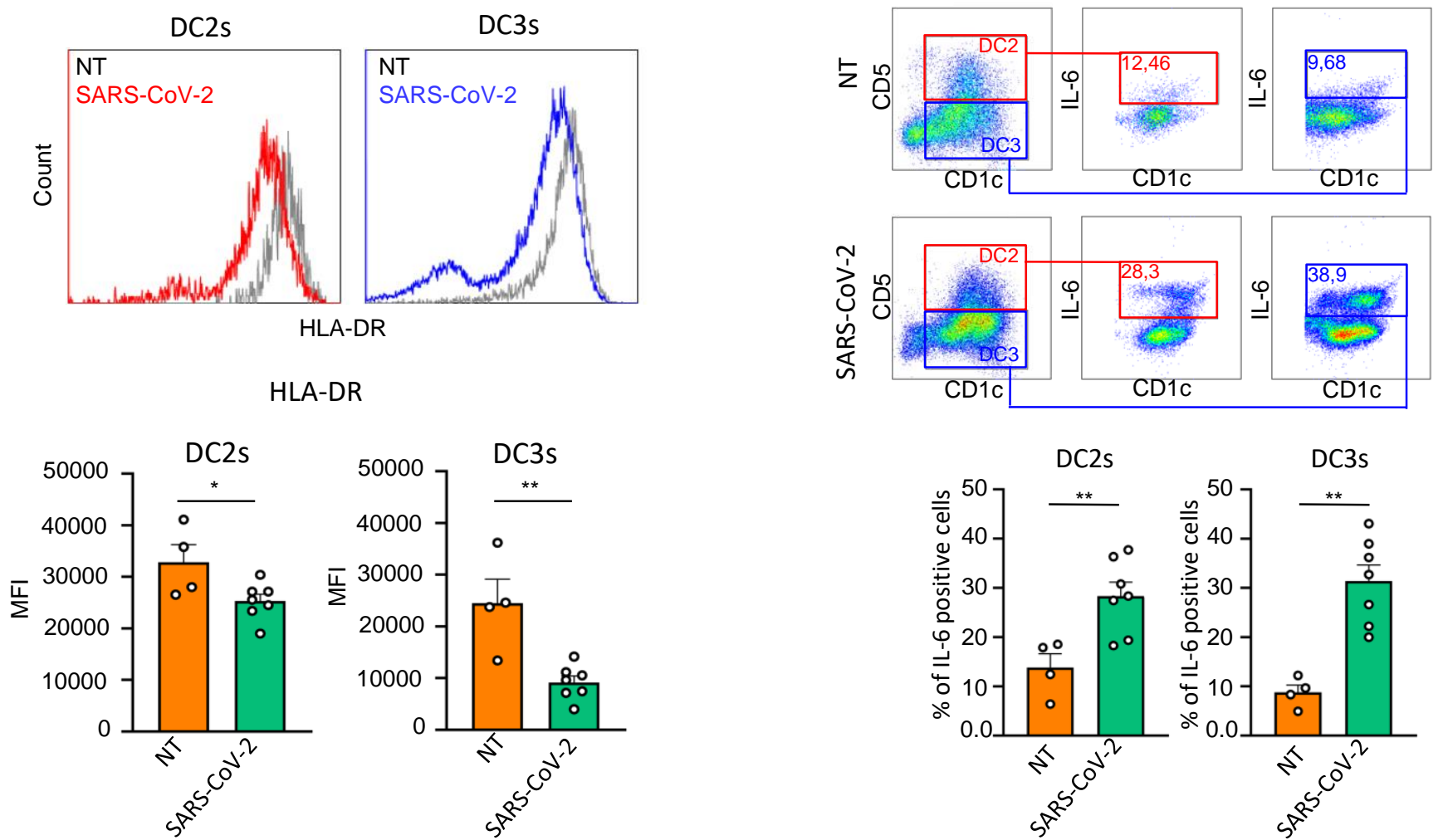

C HLA-DR expression and IL-6 production in DC2s and DC3s incubated with serum of COVID-19 patients

IL-6

DC2s

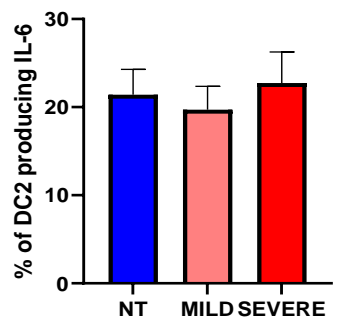

DC3s

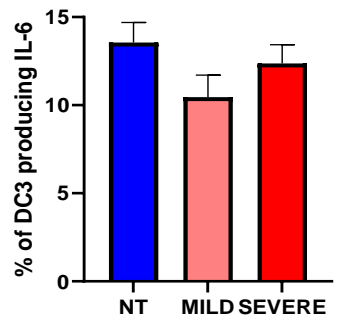

HLA-DR

DC2s

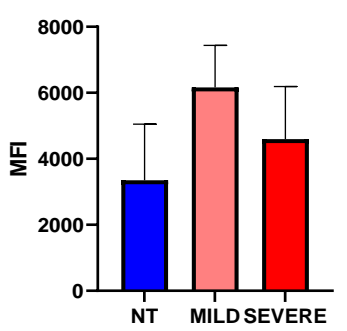

DC3s

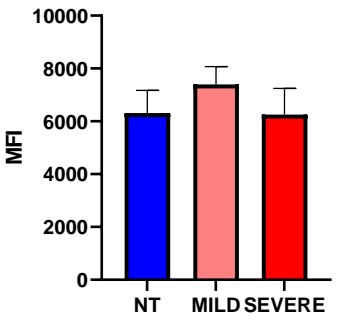

\section{Figure 5}




\section{Supplementary Figures and Tables}

A Gate strategy

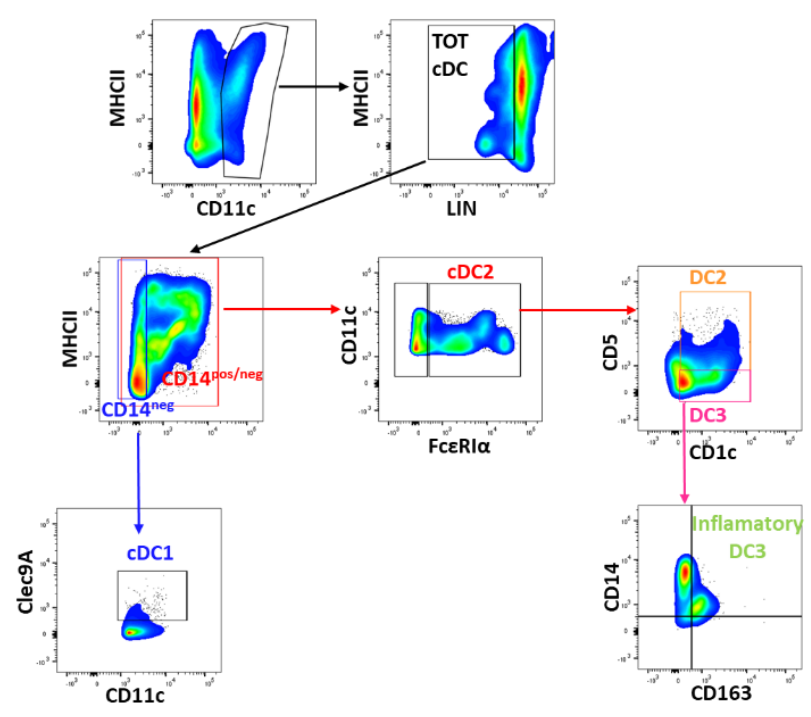

B Sorting strategy
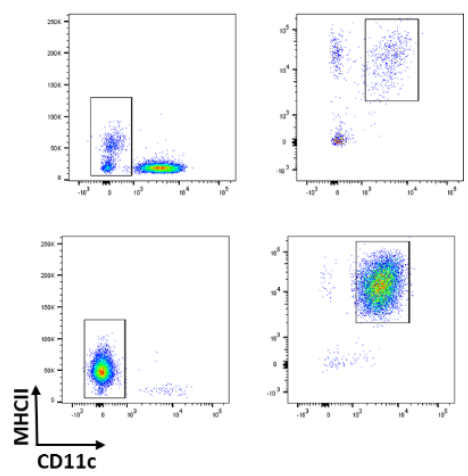

\section{Supplementary Figure 1.}

(A) Gating strategy to identify DCs subsets from PBMCs. Total DCs (cDCs TOT) were detected among the CD11 $\mathrm{c}^{+}$MHC-II ${ }^{+}$and $\mathrm{LIN}^{-}$(CD88, CD89, CD3 and CD19) population. cDC1s were identified as CLEC9A ${ }^{+}$from the CD14- fraction of total DCs. cDC2s (FceRI $\left.\alpha^{+}\right)$ include $\mathrm{CD}_{14}^{+}$and $\mathrm{CD} 14^{-}$cells. DC2s and DC3s were identified as $\mathrm{CD}^{+} \mathrm{CD}^{+} \mathrm{c}^{+}$and $\mathrm{CD}^{-}$ $\mathrm{CD} 1 \mathrm{c}^{+}$respectively. Inflammatory $\mathrm{DC} 3 \mathrm{~s}$ were recognized as $\mathrm{CD} 14^{+} \mathrm{CD} 163^{+}$cells. 
A UMAP and clustering of single cells from dataset 1

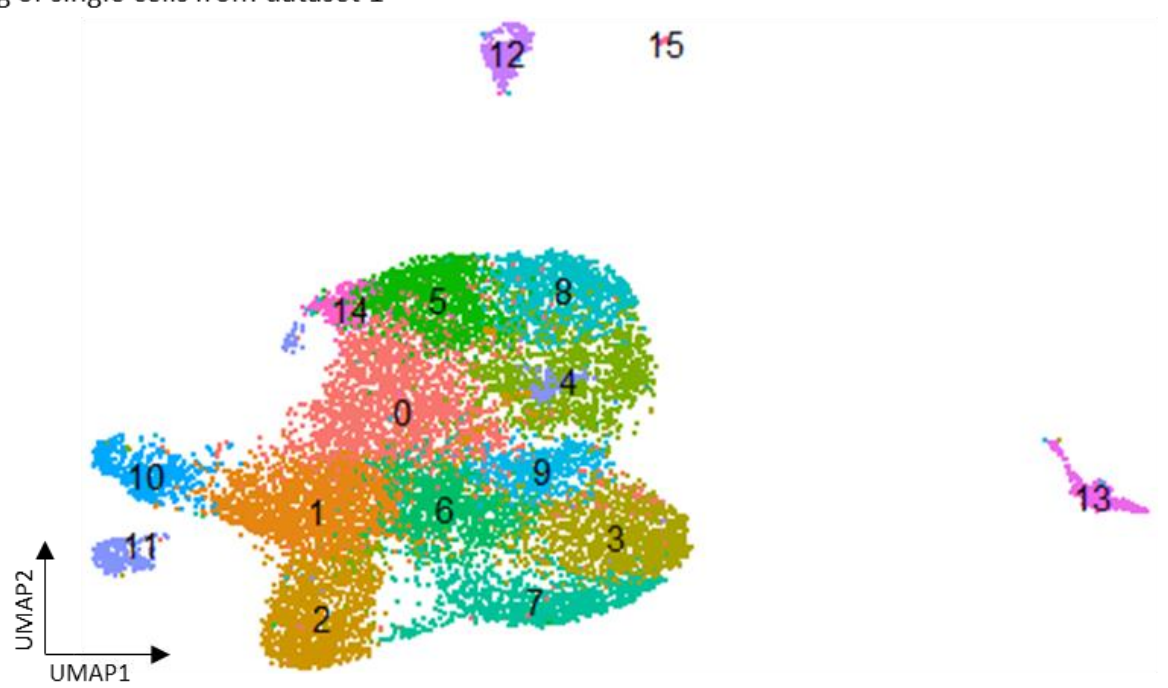

B Manual annotation of cDCs using marker genes

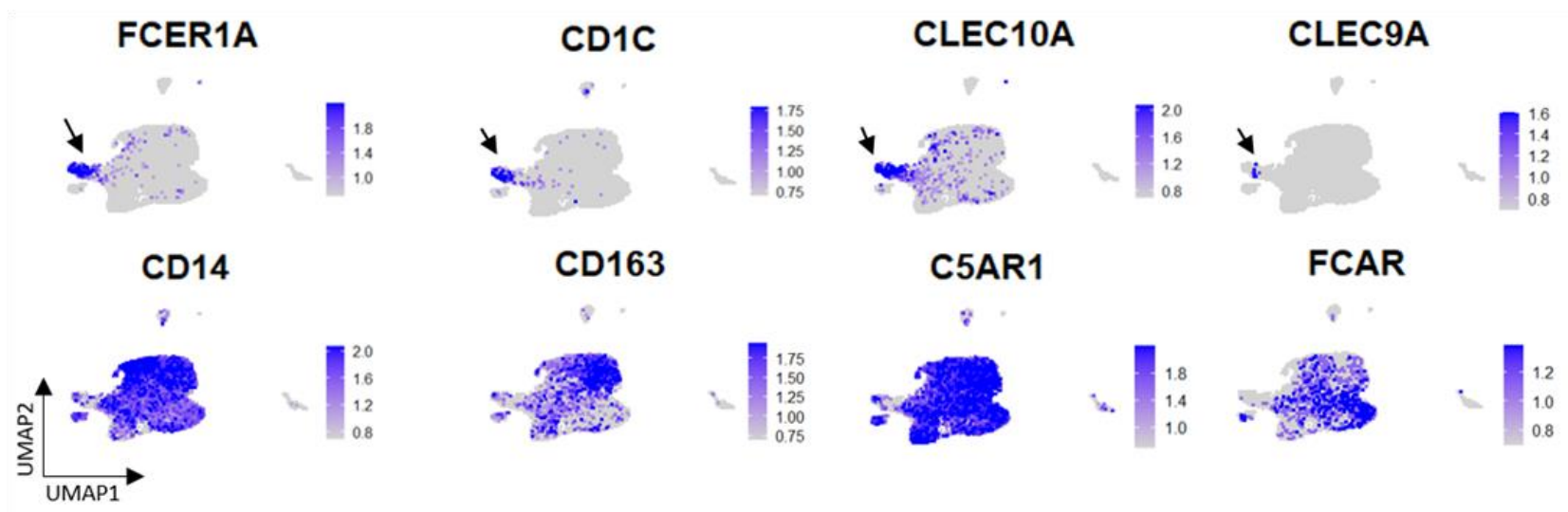

\section{Supplementary Figure 2.}

(A) UMAP and clustering of single cells from dataset 1. (B) Feature plots showing the expression levels of selected marker genes used to identify cDC cluster. Black arrows indicate cDC cluster (cluster 10). This cluster was re-clustered in a final iteration to clearly delineate cDC subsets as shown in Figure 1B. 
A UMAP and clustering of single cells from dataset 2

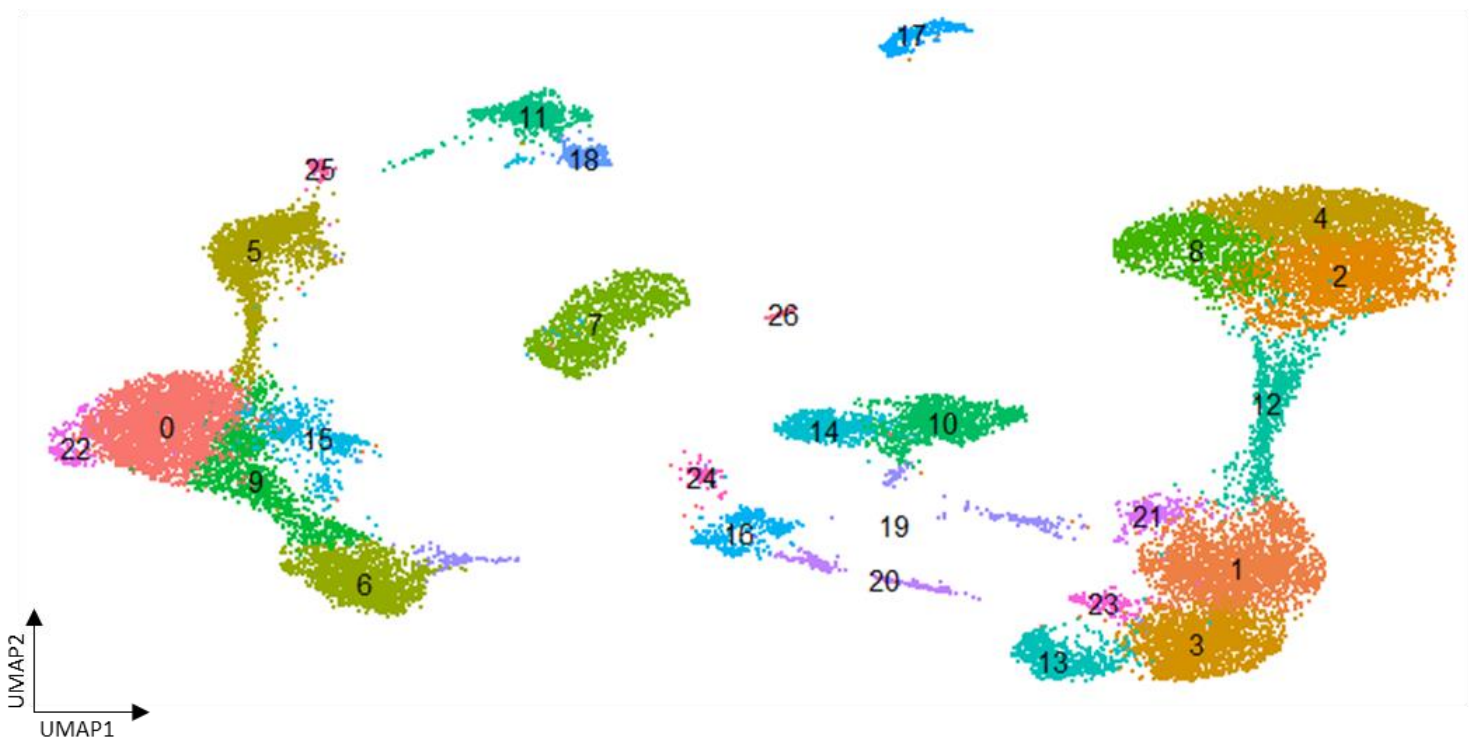

B Manual annotation of $\mathrm{cDCs}$ using marker genes

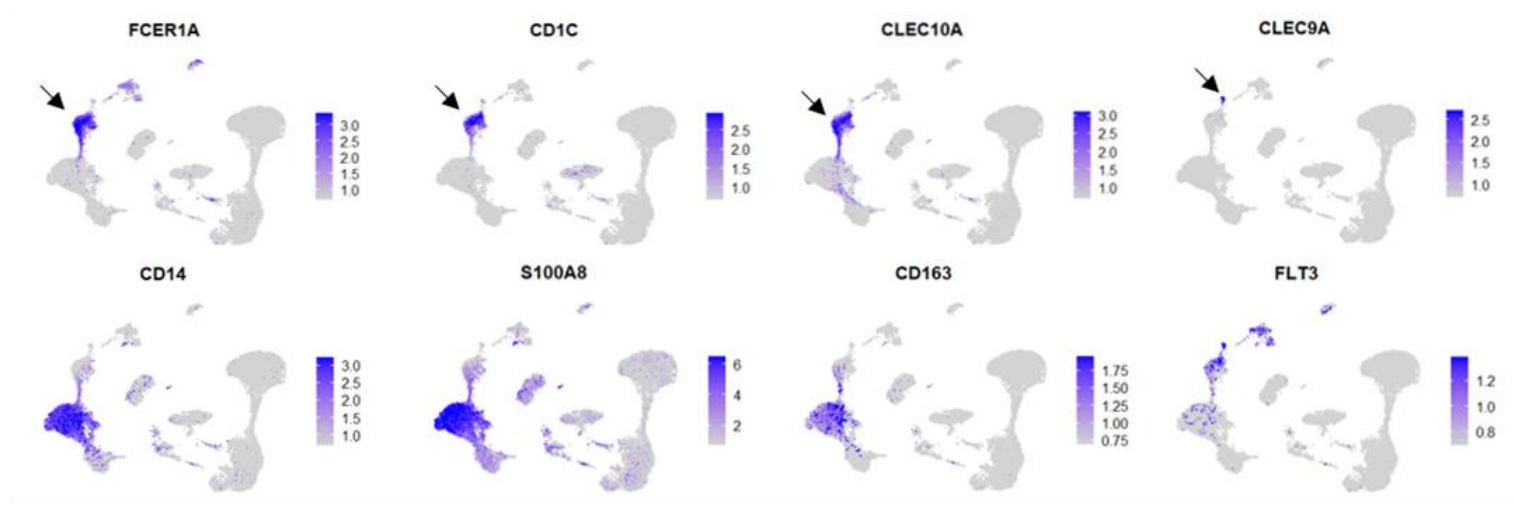

C Re-clustering of clusters 5 and 25 (cDCs)

D Violin plots referred to clusters in (C)
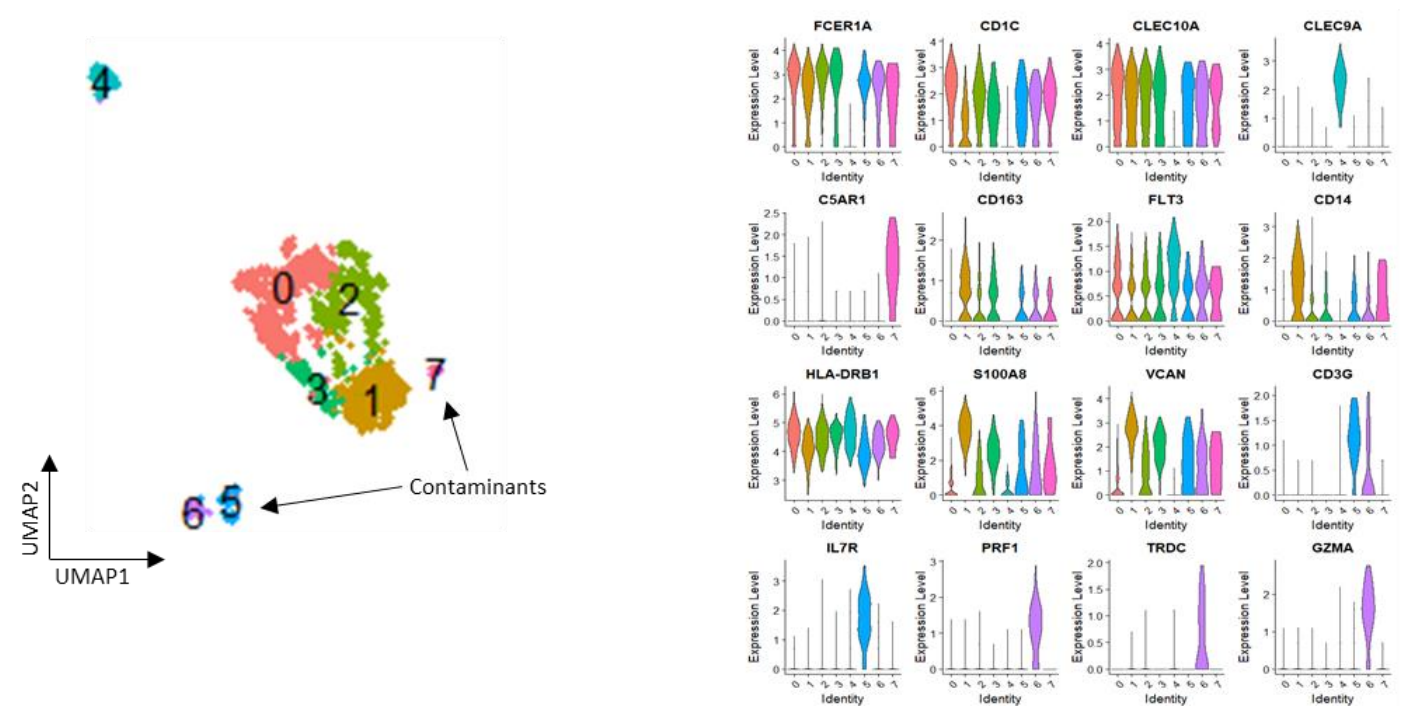

\section{Supplementary Figure 3.}

(A) UMAP and clustering of single cells from dataset 2. (B) Feature plots showing the expression levels of selected marker genes used to identify cDC clusters. Black arrows indicate 
cDC clusters (cluster 5 is cDC2 and cluster 25 is cDC1). (C) Re-clustering of clusters 5 and 25 corresponding to cDCs. Clusters 5, 6 and 7 were identified as contaminants. Clusters 0, 1, 2, 3 and 4 were re-clustered in a final iteration to clearly delineate cDC1, DC2 and DC3 subsets as shown in Figure 1B. (D) Violin plots referred to clusters in (C) showing expression levels of selected marker genes. 
A Top 100 DEGs: dataset 1

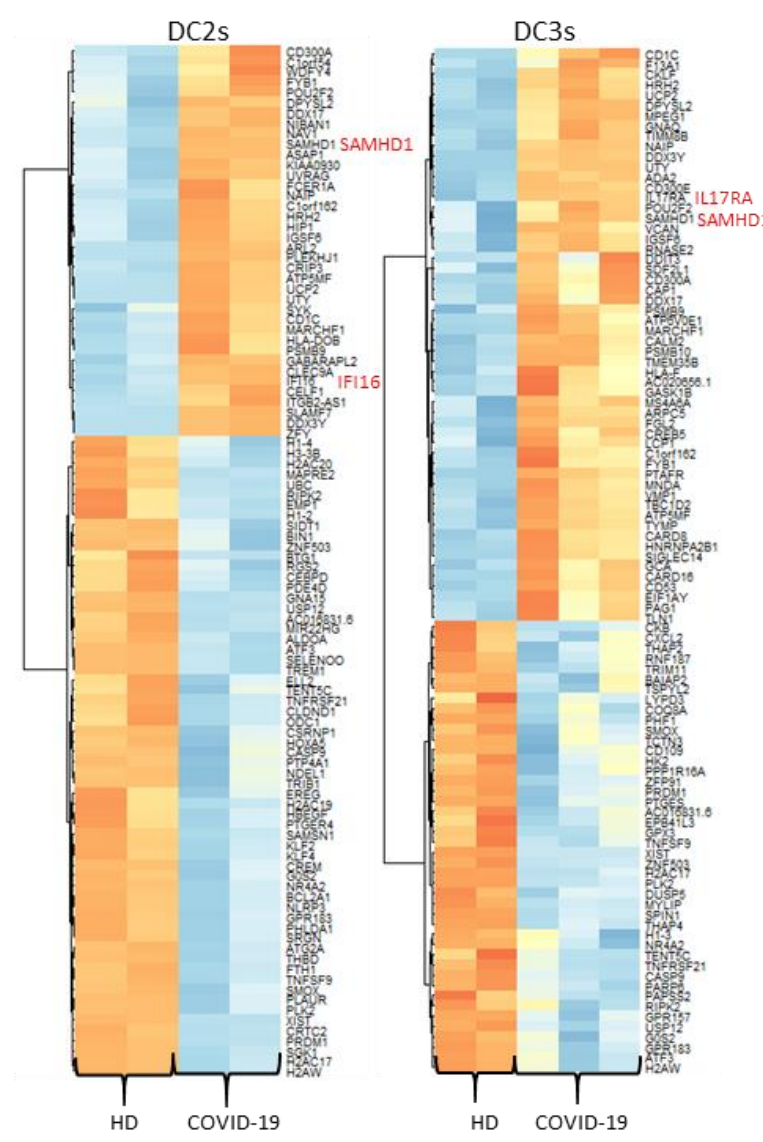

B Top 100 DEGs: dataset 3

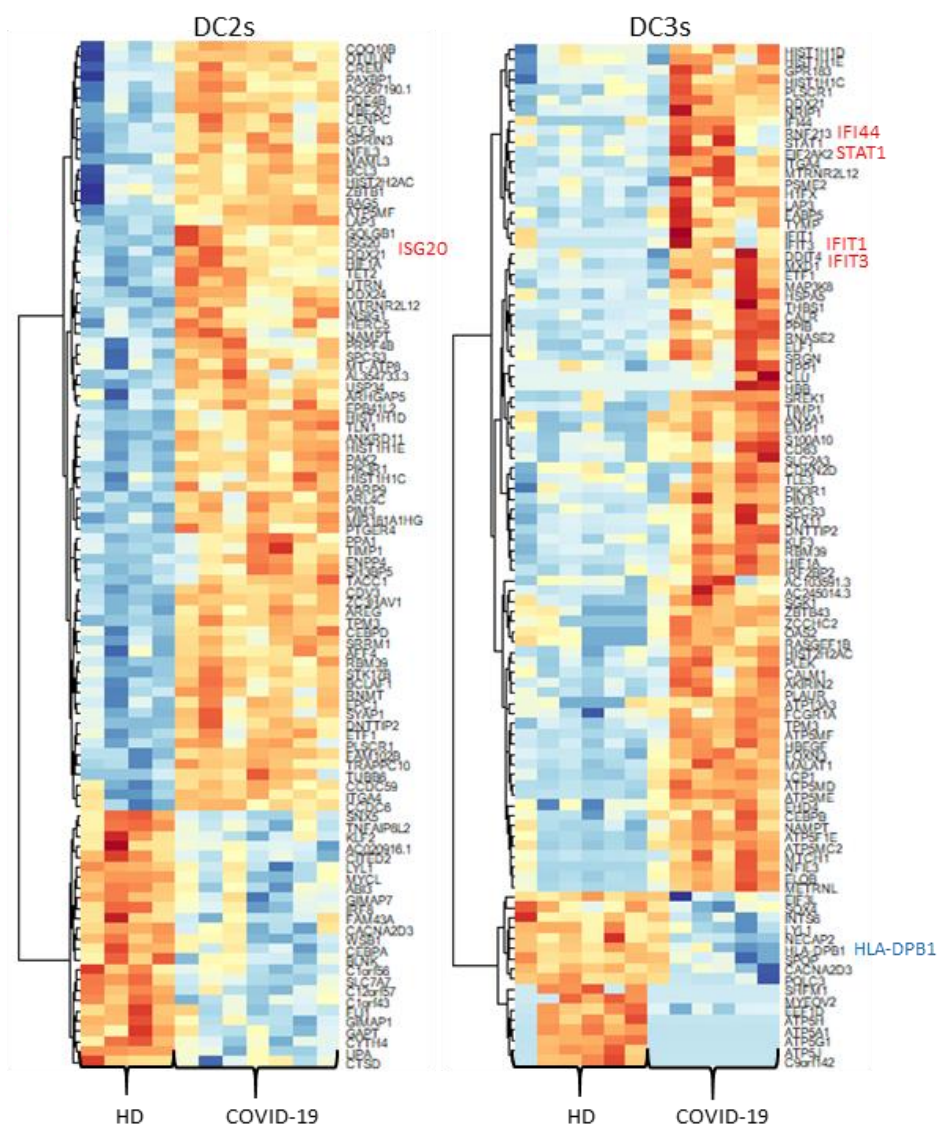

\section{Supplementary Figure 4.}

Heatmaps showing the top 100 DEGs for DC2 and DC3 subsets comparing COVID-19 patients and HDs from (A) dataset 1 and (B) dataset 2. Selected up-regulated genes are marked in red and down-regulated genes in blue. Ribosomal protein (RP) genes were removed from the top 100 DEGs. 
bioRxiv preprint doi: https://doi.org/10.1101/2021.03.03.433597; this version posted April 2, 2021. The copyright holder for this preprint (which was not certified by peer review) is the author/funder. All rights reserved. No reuse allowed without permission.

A GSEA with Blood Transcription Modules (BTM)

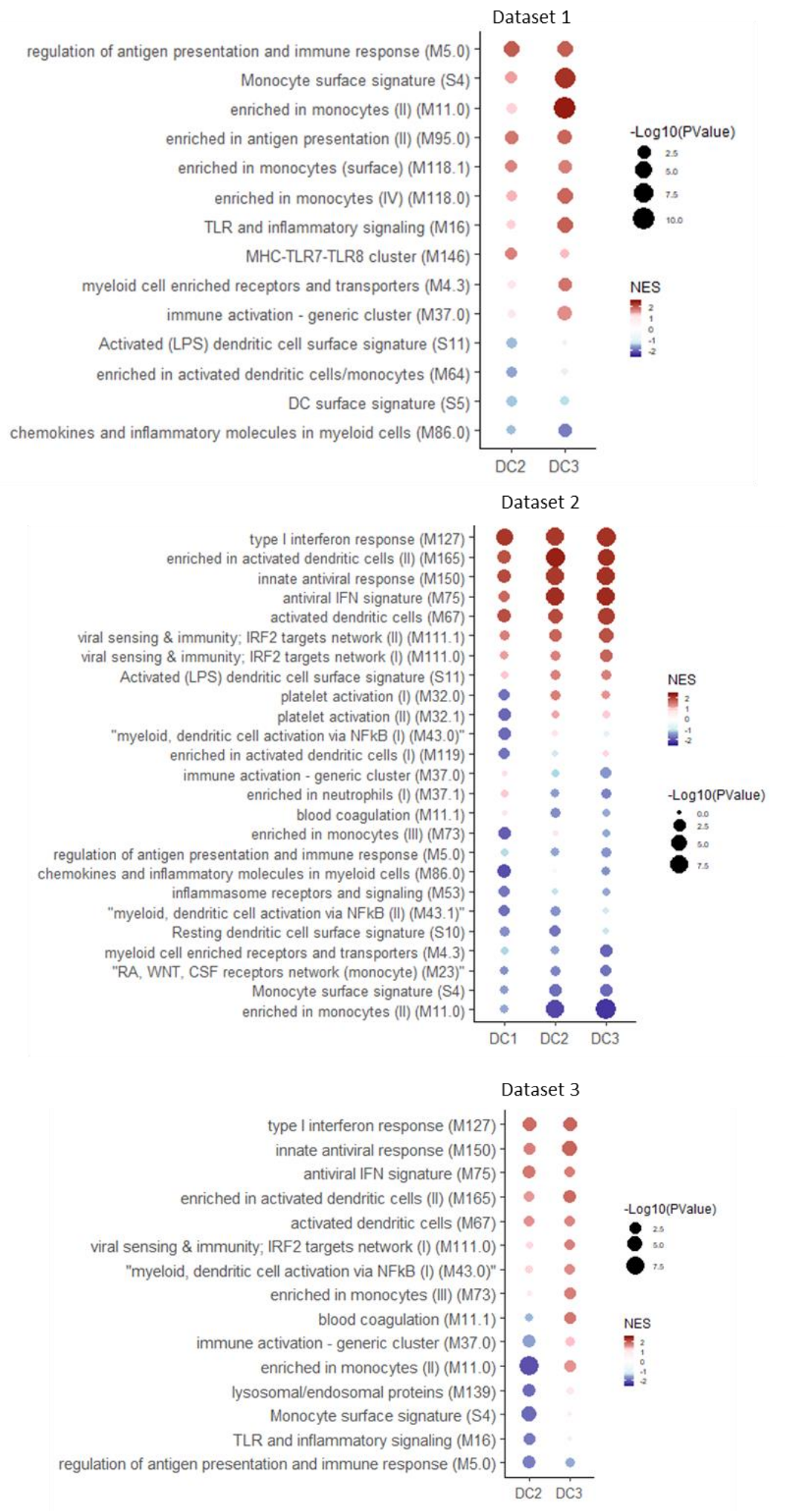

\section{Supplementary Figure 5.}

(A) GSEA of DEGs using the BTM collection: dataset 1 (upper panel), dataset 2 (middle panel) and dataset 3 (lower panel). For each DC subset, top 10 pathways based on significance are shown. NES, normalized enrichment score. 
A UMAP and clustering of cells annotated as DCs or monocytes from Reyes et al. dataset
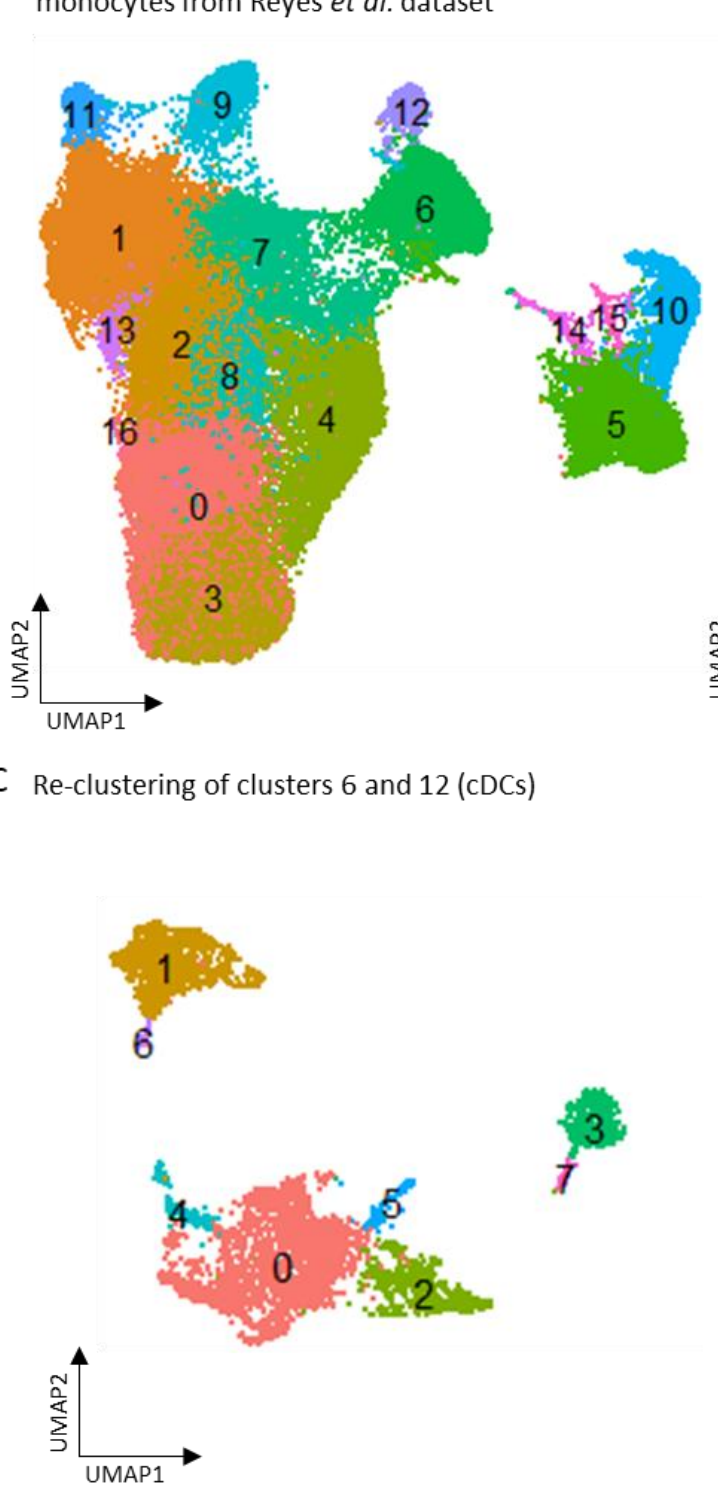

B Manual annotation of cDCs using marker genes
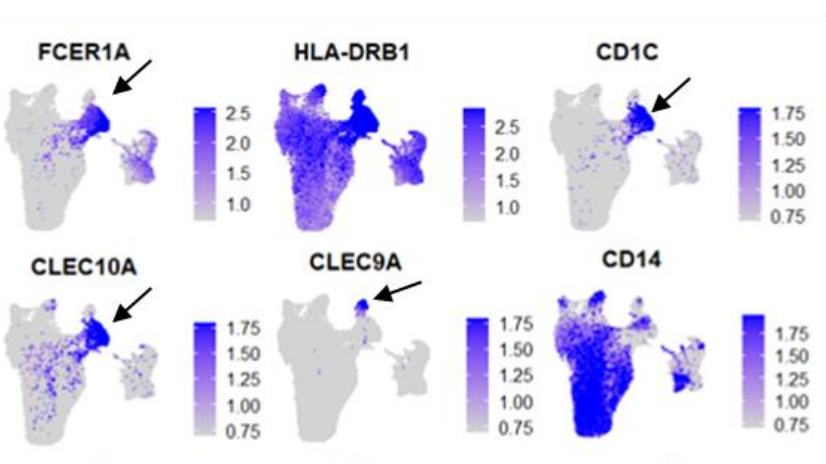

CLEC9A

4

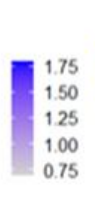

CD14

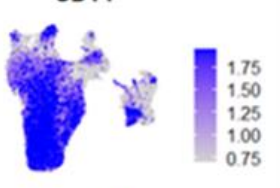

CD163 C5AR1

FCGR3A

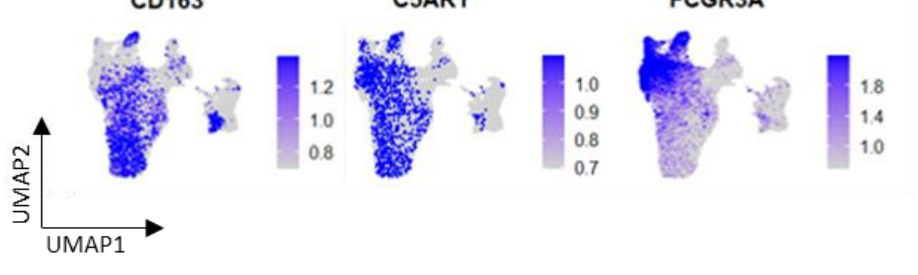

D Violin plots referred to clusters in (C)
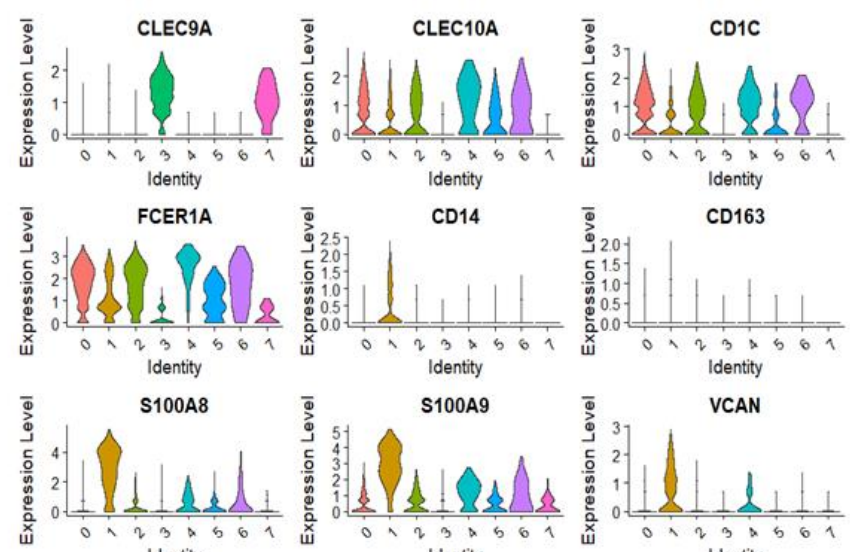

Identity
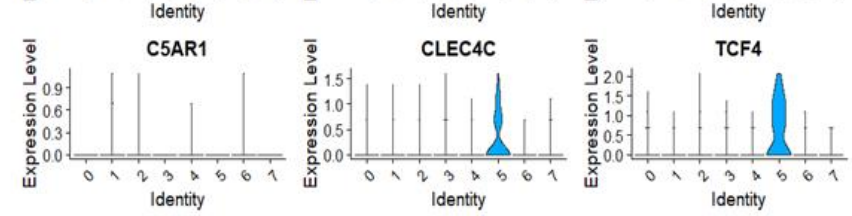

\section{Supplementary Figure 6.}

(A) UMAP and clustering of cells annotated as DCs or monocytes by the authors. (B) Feature plots showing the expression levels of selected marker genes used to identify cDC clusters. Black arrows indicate cDC clusters (cluster 6 is cDC2 and cluster 12 is cDC1). (C) Reclustering of clusters 6 and 12 corresponding to cDCs. (D) Violin plots referred to clusters in (C) showing expression levels of selected marker genes. Cluster 5, positive for CLEC4C and TCF4 was identified as contaminant and removed. All other clusters were re-clustered in a final iteration to clearly delineate cDC1, DC2 and DC3 subsets as shown in Figure 2C. 
A Top 100 DEGs: Reyes et al. dataset
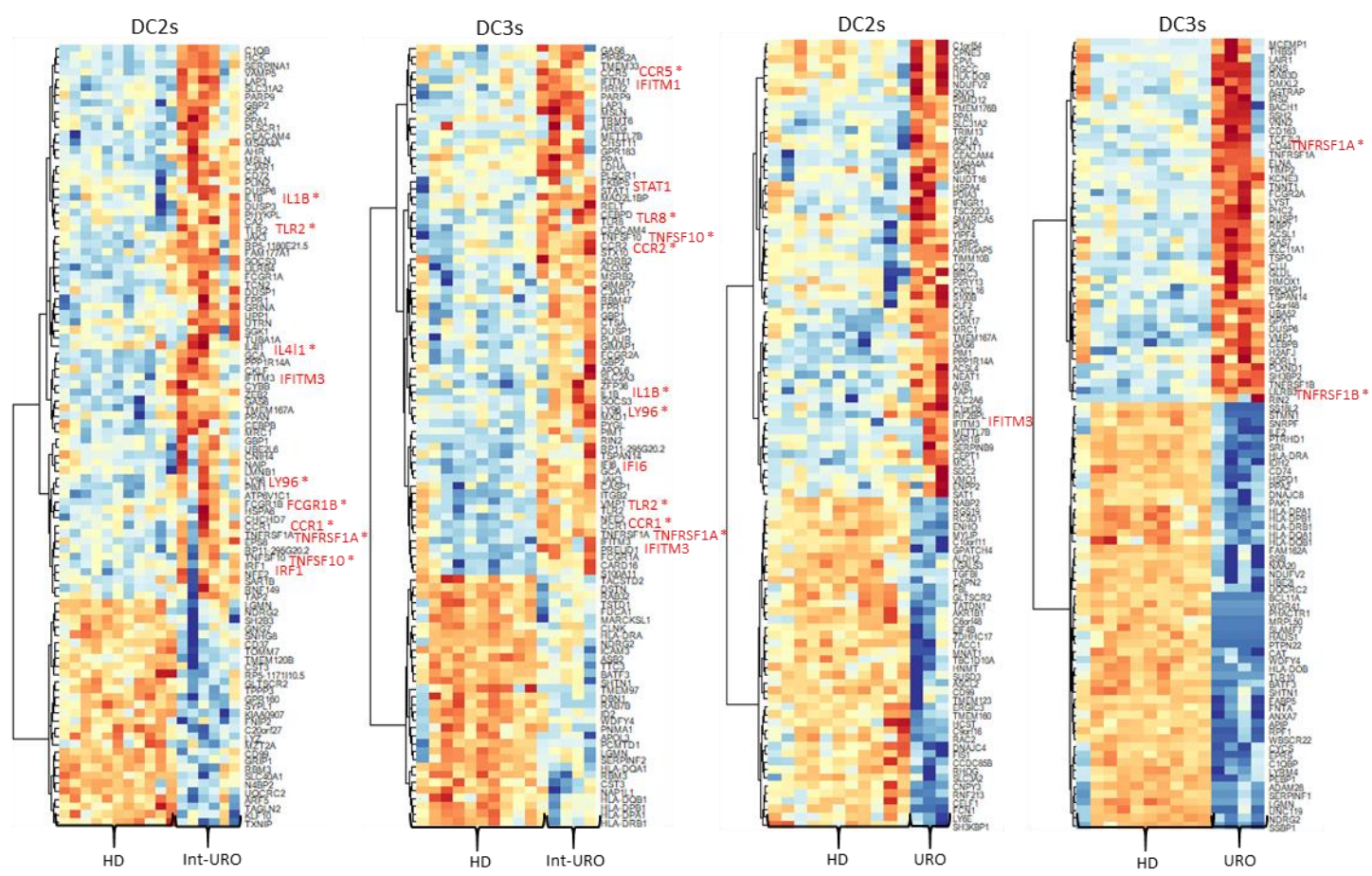

B Top 100 DEGs: Hao et al. dataset
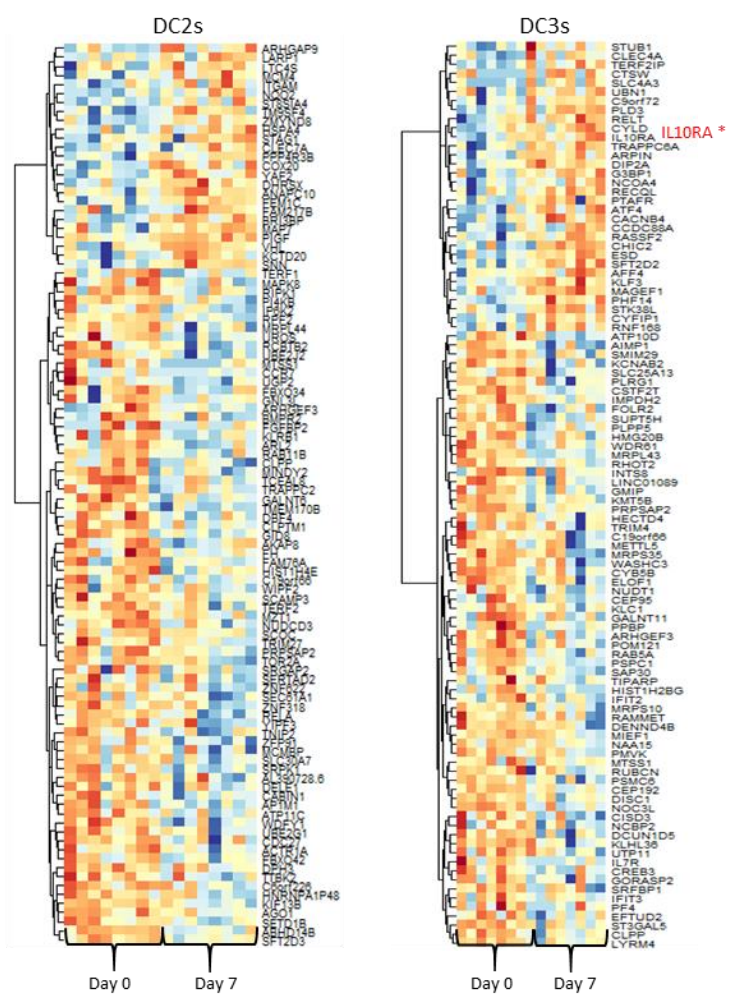

Supplementary Figure 7. Heatmaps showing the top 100 DEGs for DC2 and DC3 subsets from (A) Reyes et al. and (B) Hao et al. datasets. Selected up-regulated genes are marked in red. Asterisk indicates genes associated with pro-inflammatory functions. Ribosomal protein (RP) genes were removed from the top 100 DEGs. Int-URO, intermediate urosepsis. URO, urosepsis. 
bioRxiv preprint doi: https://doi.org/10.1101/2021.03.03.433597; this version posted April 2, 2021. The copyright holder for this preprint (which was not certified by peer review) is the author/funder. All rights reserved. No reuse allowed without permission.

A GSEA with Blood Transcription Modules (BTM): Reyes et al. dataset

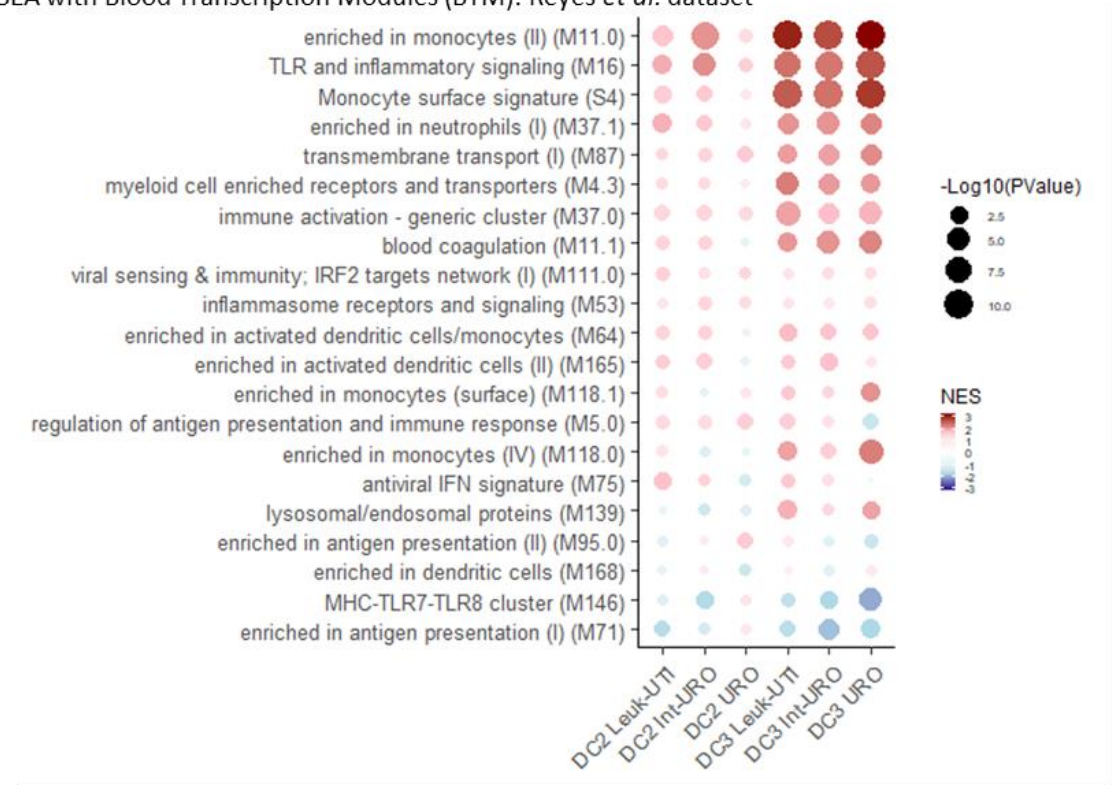

B GSEA with Blood Transcription Modules (BTM): Hao et al. dataset

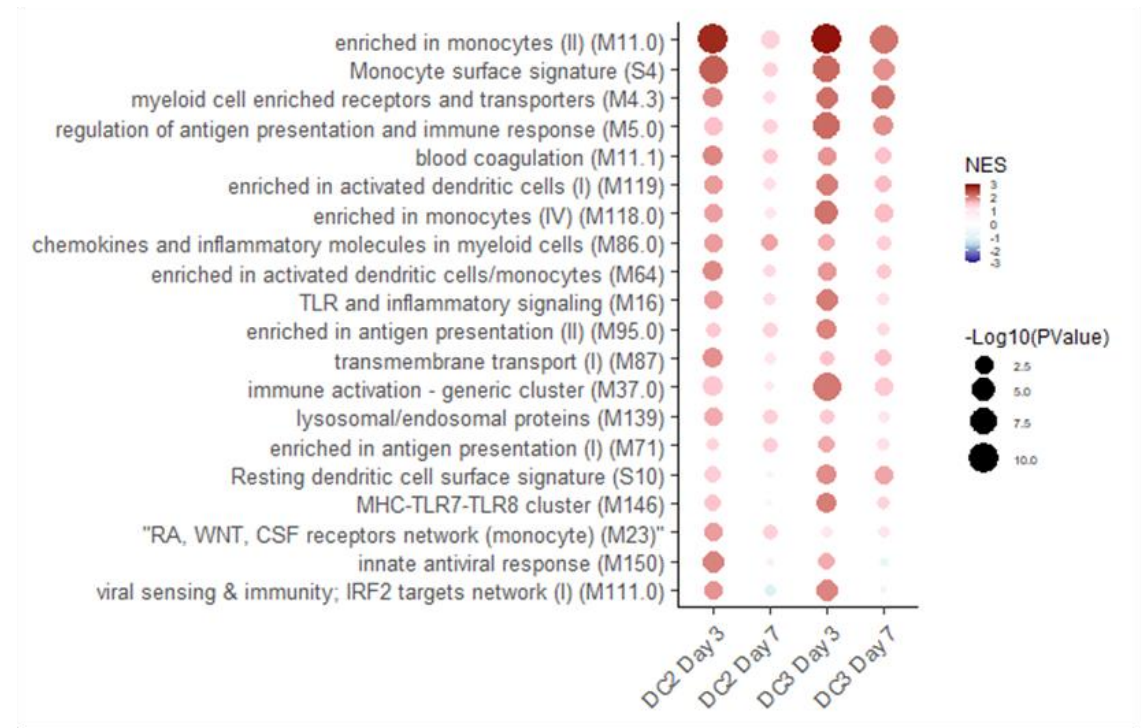

C Leading edge genes of the inflammatory response pathway: Reyes et al. dataset
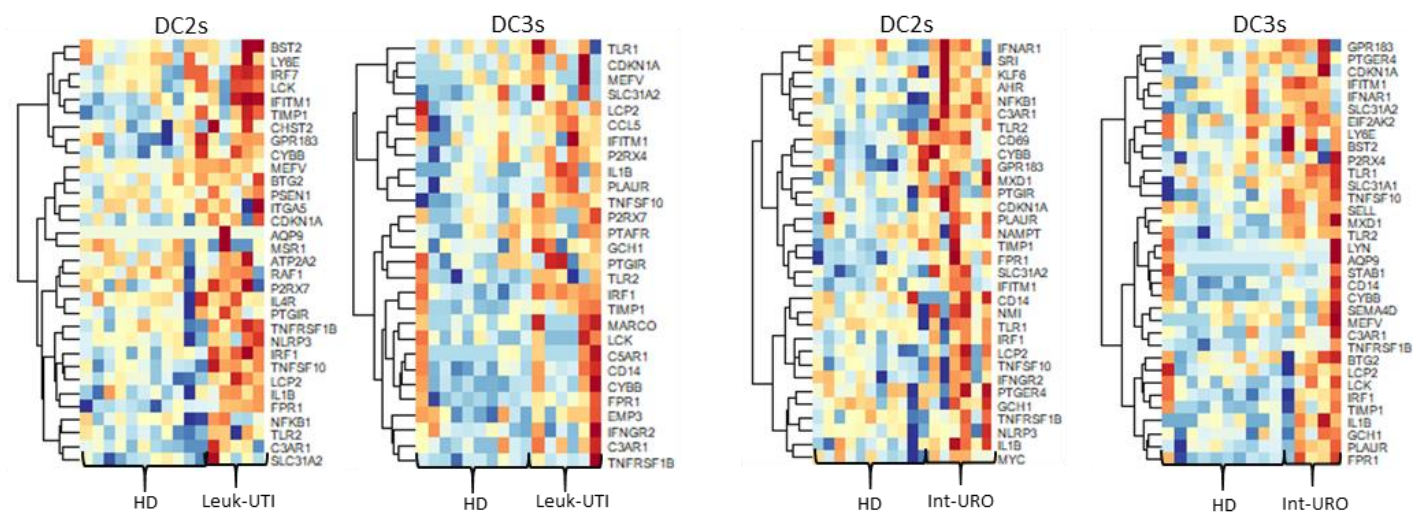

\section{Supplementary Figure 8.}

GSEA of DEGs using the BTM collection: (A) Reyes et al. dataset and (B) Hao et al. dataset. For each DC subset, top 10 pathways based on significance are shown. NES, normalized 
enrichment score. (C) Heatmaps showing leading edge genes of the inflammatory response pathway in the Reyes et al. dataset. Leuk-UTI, urinary tract infection with leukocytosis. IntURO, intermediate urosepsis. URO, urosepsis. 
Table S1. Characteristics of COVID-19 patients and healthy donors (HD) enrolled in the study.

\begin{tabular}{|c|c|c|c|c|}
\hline sample ID & Disease severity & age & gender & experiment \\
\hline Covid 1 & 5, SEVERE & 62 & female & scRNAseq \\
\hline Covid 2 & $4, \mathrm{MILD}$ & 66 & male & scRNAseq \\
\hline Covid 3 & $4, \mathrm{MID}$ & 68 & female & scRNAseq \\
\hline HD1 & $\mathrm{N} / \mathrm{A}$ & $\mathrm{N} / \mathrm{A}$ & $\mathrm{N} / \mathrm{A}$ & scRNAseq \\
\hline $\mathrm{HD} 2$ & $\mathrm{~N} / \mathrm{A}$ & $\mathrm{N} / \mathrm{A}$ & $\mathrm{N} / \mathrm{A}$ & scRNAseq \\
\hline Covid 4 & 5, SEVERE & 87 & male & DCs frequency analysis \\
\hline Covid 5 & 5, SEVERE & 68 & male & DCs frequency analysis \\
\hline Covid 6 & 5, SEVERE & 49 & male & DCs frequency analysis \\
\hline Covid 7 & 4, MILD & 79 & male & DCs frequency analysis \\
\hline Covid 8 & 6, SEVERE & 77 & male & DCs frequency analysis \\
\hline Covid 9 & 4, MILD & 60 & male & DCs frequency analysis \\
\hline Covid 10 & 4, MILD & 81 & female & DCs frequency analysis \\
\hline Covid 11 & 4, MILD & 84 & male & DCs frequency analysis \\
\hline Covid 12 & 4, MILD & 67 & female & DCs frequency analysis \\
\hline Covid 13 & 4, MILD & 69 & male & DCs frequency analysis \\
\hline Covid 14 & 4, MILD & 89 & female & DCs frequency analysis \\
\hline Covid 15 & 6, SEVERE & 59 & female & DCs frequency analysis \\
\hline Covid 16 & 3, MILD & 53 & male & DCs frequency analysis \\
\hline Covid 17 & $3, \mathrm{MILD}$ & 89 & male & DCs frequency analysis \\
\hline Covid 18 & 6, SEVERE & 61 & male & DCs frequency analysis \\
\hline Covid 19 & 4, MILD & 80 & female & DCs frequency analysis \\
\hline Covid 20 & 4, MILD & 79 & male & DCs frequency analysis \\
\hline Covid 21 & 4, MILD & 85 & female & DCs frequency analysis \\
\hline Covid 22 & 4, MILD & 59 & male & DCs frequency analysis \\
\hline Covid 23 & 3, MILD & 72 & male & DCs frequency analysis \\
\hline Covid 24 & 6, SEVERE & 62 & male & DCs frequency analysis \\
\hline Covid 25 & 3, MILD & 78 & male & DCs frequency analysis \\
\hline Covid 26 & 6, SEVERE & 68 & male & DCs frequency analysis \\
\hline Covid 27 & 3,MILD & 67 & male & DCs frequency analysis \\
\hline Covid 28 & $4, \mathrm{MILD}$ & 83 & female & DCs frequency analysis \\
\hline Covid 29 & 6, SEVERE & 31 & male & DCs frequency analysis \\
\hline Covid 30 & 4, MILD & 79 & male & DCs frequency analysis \\
\hline Covid 31 & 4, MILD & 80 & male & DCs frequency analysis \\
\hline Covid 32 & 3, MILD & 72 & male & DCs frequency analysis \\
\hline Covid 33 & 6, SEVERE & 65 & female & DCs frequency analysis \\
\hline HD3 & $\mathrm{N} / \mathrm{A}$ & 34 & female & DCs frequency analysis \\
\hline HD4 & $\mathrm{N} / \mathrm{A}$ & 32 & male & DCs frequency analysis \\
\hline HD5 & $\mathrm{N} / \mathrm{A}$ & 27 & male & DCs frequency analysis \\
\hline HD6 & $\mathrm{N} / \mathrm{A}$ & 40 & female & DCs frequency analysis \\
\hline HD7 & $\mathrm{N} / \mathrm{A}$ & $\mathrm{N} / \mathrm{A}$ & $\mathrm{N} / \mathrm{A}$ & DCs frequency analysis \\
\hline HD8 & N/A & $\mathrm{N} / \mathrm{A}$ & $\mathrm{N} / \mathrm{A}$ & DCs frequency analysis \\
\hline HD9 & $\mathrm{N} / \mathrm{A}$ & N/A & $\mathrm{N} / \mathrm{A}$ & DCs frequency analysis \\
\hline HD10 & $\mathrm{N} / \mathrm{A}$ & $\mathrm{N} / \mathrm{A}$ & $\mathrm{N} / \mathrm{A}$ & DCs frequency analysis \\
\hline HD11 & $\mathrm{N} / \mathrm{A}$ & $N / A$ & $N / A$ & DCs frequency analysis \\
\hline HD12 & $\mathrm{N} / \mathrm{A}$ & $\mathrm{N} / \mathrm{A}$ & $\mathrm{N} / \mathrm{A}$ & DCs frequency analysis \\
\hline HD13 & $\mathrm{N} / \mathrm{A}$ & $N / A$ & $\mathrm{~N} / \mathrm{A}$ & DCs frequency analysis \\
\hline HD14 & $\mathrm{N} / \mathrm{A}$ & 57 & female & DCs frequency analysis \\
\hline HD15 & $\mathrm{N} / \mathrm{A}$ & 34 & male & DCs frequency analysis \\
\hline HD16 & $\mathrm{N} / \mathrm{A}$ & 41 & male & DCs frequency analysis \\
\hline HD17 & $\mathrm{N} / \mathrm{A}$ & 85 & male & DCs frequency analysis \\
\hline HD18 & $\mathrm{N} / \mathrm{A}$ & 62 & $\mathrm{~N} / \mathrm{A}$ & DCs frequency analysis \\
\hline HD19 & N/A & 73 & $\mathrm{~N} / \mathrm{A}$ & DCs frequency analysis \\
\hline $\mathrm{HD} 20$ & $\mathrm{~N} / \mathrm{A}$ & 57 & $\mathrm{~N} / \mathrm{A}$ & DCs frequency analysis \\
\hline $\mathrm{HDZ}$ & $\mathrm{N} / \mathrm{A}$ & 34 & $\mathrm{~N} / \mathrm{A}$ & DCs frequency analysis \\
\hline $\mathrm{HD} 22$ & $\mathrm{~N} / \mathrm{A}$ & 70 & $\mathrm{~N} / \mathrm{A}$ & DCs frequency analysis \\
\hline $\mathrm{HD} 23$ & $\mathrm{~N} / \mathrm{A}$ & 64 & $\mathrm{~N} / \mathrm{A}$ & DCs frequency analysis \\
\hline
\end{tabular}


Table S2. DEGs between COVID-19 patients and healthy donors in each DC subset from datasets 1, 2 and 3.

Table S3. BTM families used for GSEA.

Table S4. DEGs in each DC subset from Reyes et al. and Hao et al. datasets.

Table S5. DEGs in DC3s compared with DC2s in response to SARS-CoV-2 infection and intermediate urosepsis.

Table S6. DEGs in DC2s and DC3s in severe compared with mild patients. 